\title{
Q1 Reading visually embodied meaning from the brain: Visually grounded computational models decode visual-object mental imagery induced by written text
}

\author{
22 Andrew James Anderson ${ }^{\mathrm{a}, \mathrm{b}, *}$, Elia Bruni ${ }^{\mathrm{b}}$, Alessandro Lopopolo ${ }^{\mathrm{b}}$, Massimo Poesio ${ }^{\mathrm{b}, \mathrm{c}}$, Marco Baroni $^{\mathrm{b}}$ \\ a Brain and Cognitive Sciences, University of Rochester, NY 14627, USA \\ b Center for Mind/Brain Sciences, 38068, Rovereto, Italy \\ c University of Essex, CO4 3SQ, UK
}

\section{A R T I C L E I N F O}

\section{Article history:}

Received 26 November 2014

Accepted 30 June 2015

Available online $\mathrm{xxxx}$

\section{Keywords:}

Concept representation

Embodiment

Mental imagery

Perceptual simulation

Language

Multimodal semantic models

Representational similarity

\begin{abstract}
A B S T R A C T
Embodiment theory predicts that mental imagery of object words recruits neural circuits involved in object per- 21 ception. The degree of visual imagery present in routine thought and how it is encoded in the brain is largely un- 22 known. We test whether fMRI activity patterns elicited by participants reading objects' names include embodied 23 visual-object representations, and whether we can decode the representations using novel computational image- 24 based semantic models. We first apply the image models in conjunction with text-based semantic models to test 25 predictions of visual-specificity of semantic representations in different brain regions. Representational similarity 26 analysis confirms that fMRI structure within ventral-temporal and lateral-occipital regions correlates most 27 strongly with the image models and conversely text models correlate better with posterior-parietal/lateral-tem- 28 poral/inferior-frontal regions. We use an unsupervised decoding algorithm that exploits commonalities in repre- 29 sentational similarity structure found within both image model and brain data sets to classify embodied visual 30 representations with high accuracy (8/10) and then extend it to exploit model combinations to robustly decode 31 different brain regions in parallel. By capturing latent visual-semantic structure our models provide a route into 32 analyzing neural representations derived from past perceptual experience rather than stimulus-driven brain ac- 33 tivity. Our results also verify the benefit of combining multimodal data to model human-like semantic 34 representations.
\end{abstract}

(c) 2015 Published by Elsevier Inc.

\section{Introduction}

Embodiment theory predicts that visual aspects of object-related semantic knowledge are simulated in the visual system even when reading, but there is little quantitative information on the depth and spatial distribution of visual content in imagined representations. This article uses novel image-based computational models of generic concepts to distinguish visual from non-visual aspects of fMRI activity patterns cued by reading object nouns, without the participant having been explicitly asked to mentally visualize specific object images (as in previous studies classifying mental imagery e.g. Lee et al., 2012; Reddy et al., 2010) and quantitatively demonstrates detailed visual imagery by decoding multiple categories from its content using the models. We target three questions:

(1) Are visually embodied object representations elicited when people read and contemplate object words?

* Corresponding author at: Brain and Cognitive Sciences, University of Rochester, NY 14627, USA.

E-mail address: andrewanderson@bcs.rochester.edu (A.J. Anderson).
(2) How are visually embodied representations distributed through- 56 out the cortex and how does this distribution relate to language- 57 based semantic representations?

(3) How detailed is the visual-object mental imagery - can our 59 models correctly assign labels to multiple object categories in un- 60 labeled brain representations?

Why might we expect reading to induce visual object representations in the 63 brain?

Building successively on behavioral evidence supporting both Paivio Q3 (1971) dual-coding theory and Glaser's (1992) lexical hypothesis theo- 66 ry, behavioral/fMRI evidence for Barsalou et al.'s (2008) language and 67 situated simulation theory, recent EEG results support the view that 68 word comprehension involves initial activation of a shallow language- 69 based conceptual representation (for rapid semantic evaluation) that 70 is later complemented by a deeper simulation of the visual properties 71 of the concept (Louwerse and Hutchinson, 2012). More generally 72 speaking an increasing body of evidence supports the notion that 73 
conceptual representations are embodied in sensory and motor systems, as opposed to being purely language-based (recent reviews are Binder and Desai, 2011; Pulvermüller, 2013). Conceptual representations of object words (animals, tools) are associated to brain regions linked to visual object perception (Martin, 2007), reading the names of objects that have acoustic properties (telephone) activates auditory processing regions (Kiefer et al., 2008) and reading action words elicits activity in representations of the body (e.g., kick activates foot/legrelated brain regions, Hauk et al., 2004). Beyond this relation between object words and perceptual/motor brain regions linked to experiencing the objects, little is known about the nature of embodied representations. Are they internally synthesized in some detail as a valuable component of cognition (Barsalou, 2009; Pulvermüller et al., 2010; Trumpp et al., 2013) or is perceptual and/or motor overlap a nonessential epiphenomena of thought (Bedny and Caramazza, 2011)? Regarding the depth of simulation Zwaan et al. (2002) observe behavioral differences consistent with perceptual representations of the overall shape of objects being automatically activated in sentence comprehension and Kellenbach et al. (2000) observe associated EEG signal differences using a perceptual semantic priming task. That detailed visually embodied aspects of concept representations elicited in language tasks can be extracted from fMRI data has not previously been demonstrated.

Why might we expect to identify detailed visually embodied representations in fMRI concepts?

Building on pioneering fMRI studies (Ishai et al., 2000; O'Craven et al., 1999), several recent multivariate analyses targeting relationships between visual perception and visual mental imagery (Lee et al., 2012; Reddy et al., 2010; Stokes et al., 2009a; Stokes et al., 2011)/visual attention (Peelen et al., 2009; Stokes et al., 2009b) provide evidence that internally induced visual object representations can be distinguished. More specifically, Stokes et al. (2009a), Reddy et al. (2010), Stokes et al. (2011), and Lee et al. (2012) demonstrate that fMRI activity evoked by explicit mental visualization of shape/ object images can be discriminated by classifiers trained on fMRI data recorded when the respective images were perceived. Imageperception driven fMRI object representations correlate with computer-vision models of the specific experimental images (Leeds et al., 2013), which adds to the argument that mental-imagery representations discriminated in similar brain regions in the previous studies are indeed visually grounded. Peelen et al. (2009) suggest the existence and approximate anatomical location of objectcategory-specific abstract visual templates invoked in natural scene categorization that are "invariant to geometric and photometric changes and spatially unspecific". As such visual templates must be regularly, rapidly and efficiently evoked in routine activities, it is reasonable that they would also be recruited in cognitive processing that is not driven by sensory input.

\section{How do we detect visually embodied object representations in fMRI activity} patterns?

We introduce a new method to probe embodied representations with computational semantic models. By piggybacking on extensive research in computer vision, we build image-based models of generic object representations derived from natural image statistics, and use them to search for brain regions encoding similar information. Specifically, our approach is grounded on the argument that representational similarity structure (see Kriegeskorte et al., 2008a) between image-based models and visually embodied brain representations should correlate (better than other aspects of brain representation and better than with non-visual models).
We construct image-based models through a computational proce- 135 dure (schematically illustrated in Fig. 1) that links object names to com- 136 binations of abstract visual features automatically extracted from large 137 collections of non-curated images (Bruni et al., 2014; Sivic and 138 Zisserman, 2003). The images are non-curated in sense that they have 139 not been picked nor edited to be particularly representative of the 140 depicted objects: they are pictures that were independently taken for 141 other purposes, retrieved from the internet using the object name as 142 search term, and only filtered out if they don't contain the object. How- 143 ever, the object can be occluded, it might occur in multiple instances, it 144 might be only present in the background, etc. In this sense, it is a very 145 "natural" data set. Fig. 2 provides examples of the visual features that 146 our algorithm spots and uses. Since the source images are natural pic- 147 tures capturing widely differing instances of the same object (Fig. 3), 148 we incorporate real-world levels of visual variability.

What is the difference between our image-based models and those previ- 150 ously applied?

Although image-based models have previously been used in multi- 152 voxel pattern analysis studies, unlike our generic object representations, 153 they have all been stimulus-specific, and used to track visual processing 154 of a pictorial/text stimuli (e.g., Connolly et al., 2012; Devereux et al., 155 2013; Hiramatsu et al., 2011; Kriegeskorte et al., 2008a; Leeds et al., 156 2013). These have been models of relatively low-level visual processing 157 (in particular V1 and V4, from the HMAX algorithm of Serre et al., 2007), 158 with the exception of Leeds et al. (2013) and Khaligh-Razavi and 159 Kriegeskorte (2014) who used various contemporary computer vision 160 object recognition algorithms to build stimulus-specific image-based 161 models, and our pilot analysis of Mitchell et al.'s (2008) image/text 162 cued fMRI data with prototype models that did not differentiate imag- 163 ery from other aspects of semantic representation (Anderson et al., 164 2013).

How can we distinguish visually embodied object representations from oth- 166 er aspects of embodied and disembodied representation?

Our image-based models provide a means to spot visually grounded 168 representations. However, as language can describe similarities/differ- 169 ences between visual patterns (bananas and lemons are yellow and $\mathrm{cu}-170$ cumbers are green), and indeed, due to the tendency of words to co- 171 vary with the percepts they denote, sensorial knowledge is deeply em- 172 bedded in language (Connell and Lynott, 2014; Louwerse, 2008), the 173 models risk detecting visual aspects of language-based brain patterns. 174 To deal with this we introduce a strategy where image-based models 175 are used in combination with text-based semantic models to interpret 176 fMRI activity patterns. These text-based models, induced from patterns 177 of co-occurrence of words in large text corpora, are extensively used in 178 cognitive and computer science as proxies to the linguistic aspects of 179 human semantic memory (Landauer and Dumais, 1997; Lund and 180 Burgess, 1996; Turney and Pantel, 2010). Thus, they should capture 181 those aspects of conceptual similarity that people could extract by sta- 182 tistical generalization over their linguistic experience. Mitchell et al. 183 (2008) demonstrated that such text-based semantic models encode 184 linguistically-based semantic information sufficient to predict widely 185 distributed activity patterns elicited when people think about object 186 nouns (see section on Computational text-based semantic models for 187 details about our text-based model and e.g. Devereux et al., 2010; Q5 Murphy et al., 2012; for a comparison of different text-based models ap- 189 plied to neural decoding). If there is a significant difference in correla- 190 tion strength between a brain region and the image- or text-based 191 model, it suggests that semantic representation in that region is visual- 192 ly/linguistically dominated (even if both models significantly correlate 193 with it, because of the systematic word-percept covariance patterns 194 


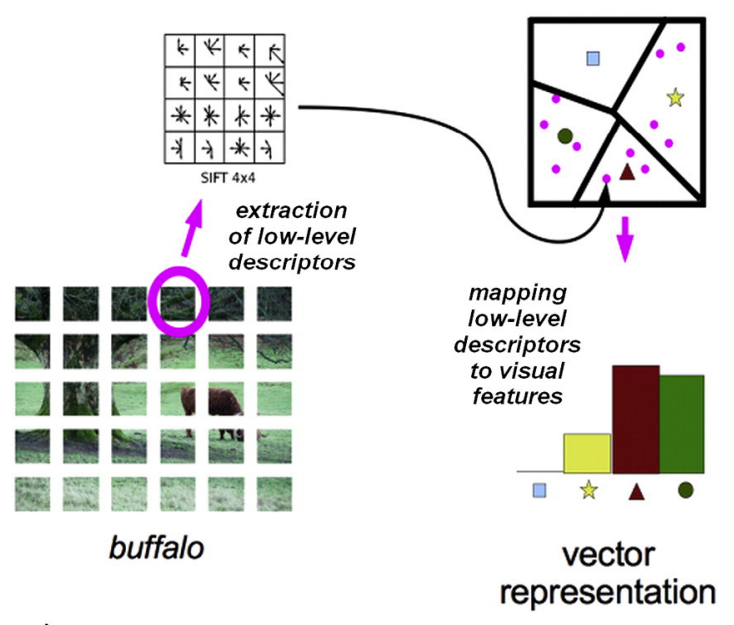

a)

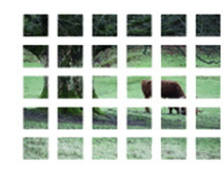

buffalo

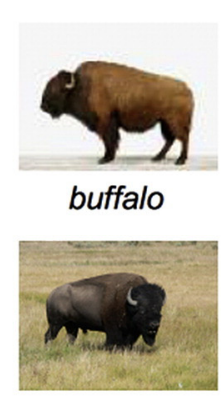

buffalo

b) we mentioned above). In particular, if a region correlates significantly more strongly with the visual model, we can be reasonably confident that it is sensitive to visual imagery information beyond what might be encoded in language. With only two models, the technique still does not safeguard against detecting embodied representations in other modalities. Since we have not developed motor/audio/smell/ etc.-based computational models yet, we deal with this risk by taking into account prior knowledge of brain region modal-specificity through specific hypothesis tests, e.g., image-based models should correlate best with brain representations in known visual processing areas, and textbased models with linguistic/amodal brain regions. We make no claim about correlation in brain areas strongly linked to non-visual modalities.

Having detected visually embodied objects how can we quantify representational detail?

If we can discriminate object categories in visually embodied neural representations, we have proof of principle that imagery in a verbal task, whether it is conscious or unconscious, contains at least category-level visual detail (and classification accuracy provides a simple metric of this detail). This in turn would suggest that category-based decision making relying on internally simulated visual properties (e.g. "which image-category would look best...") is at least a possibility, which would extend beyond the view that grounded representations are functionally irrelevant epiphenomena of linguistic activity (though our study does not attempt to distinguish between epiphenomenal visual processing and functionally relevant processing). We use an unsupervised representational-similarity-based decoding algorithm (Raizada and Connolly, 2012) to classify visual-brain patterns using the imagebased model. In addition, in line with recent proposals about how both perceptual and linguistic evidence is characterizing conceptual knowledge (Barsalou et al., 2008), and given that the previous analyses suggest that image- and text-based models provide complementary sources of information, we tailor the algorithm for multi-model brainwide decoding. In a mixture-of-experts strategy, we use the image- based model to decode visually-specific brain regions in parallel with 228 the text-based model decoding more linguistic regions.

In summary, we present:

(1) A hypothesis-driven representational similarity analysis (RSA) 231 targeting specific brain regions and distinguishing visual/linguis- 232 tic representations by differential correlation with image/text- 233 based models.

(2) An exploratory searchlight RSA charting the spread and overlap 235 of localized correlation with image/text-based models through- 236 out the brain.

(3) Image-model driven unsupervised classification of visually em- 238 bodied representations, and image/text-model decoding of mul- 239 tiple brain regions in parallel.

Materials and methods

fMRI experiment

We re-analyzed fMRI data for a popular set of stimuli (Table 1) 244 consisting of 60 concrete nouns belonging to 12 taxonomic categories 245 (because of limitations in the ImageNet database we use to construct 246 our image-based computational model, we had coverage for 51 words 247 and 11 classes) originally recorded by Just et al. (2010), but also forming 248 the basis of, e.g., Mitchell et al. (2008); Leeds et al. (2013). Eleven 249 consenting adults (consent approved by the University of Pittsburgh 250 and Carnegie Mellon Institutional Review Boards), eight female, all 251 right handed and all from the Carnegie Mellon community were 252 shown each word six times over, and were asked to actively think 253 about the properties of the object to which the word referred as further 254 described below. Just et al.'s (2010) original experiment demonstrated 255 latent, ecologically interpretable and spatially distributed semantic di- 256 mensions in the brain activity of participants thinking about the 257 nouns. Details of data collection and preprocessing are as follows. 


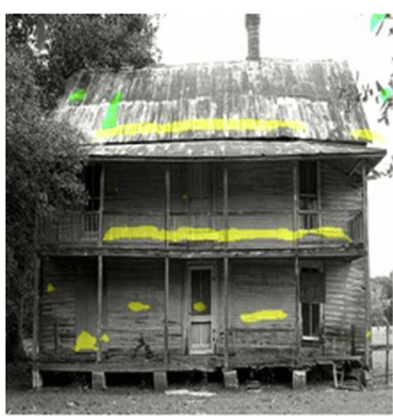

a)

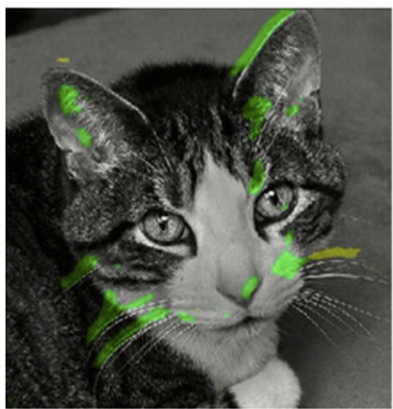

c)

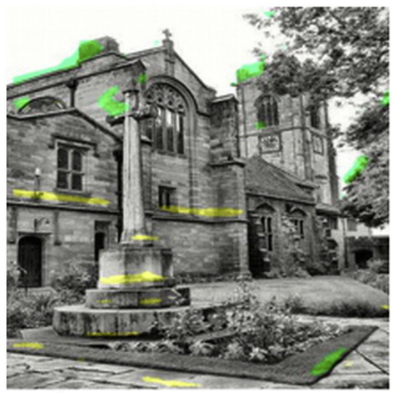

e)

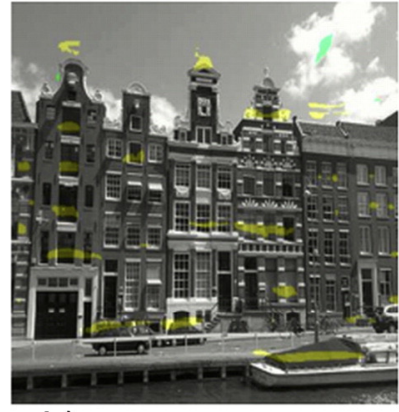

b)

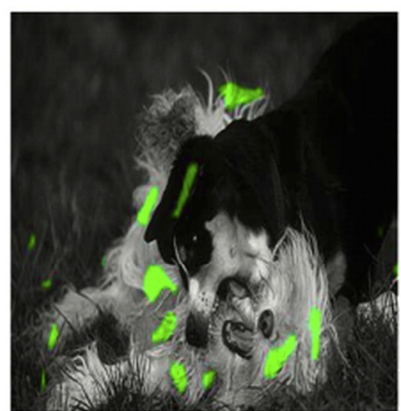

d)

Fig. 2. Backprojection of visual features mainly associated to horizontal (yellow) and curvy/oblique (green) lines.

\section{Experimental paradigm and task}

The sequence of words was presented in a random order in six runs.

Individual words were displayed for $3 \mathrm{~s}$, followed by a $7 \mathrm{~s}$ rest, during which time a fixation cross was shown. The fixation cross was also displayed 12 extra times for periods of $31 \mathrm{~s}$ to give a baseline.

On word presentation, participants actively thought about the properties of the object to which the word referred. To prompt consistent responses across all presentations of the same word participants had previously listed a set of word properties that they personally and freely associated with the noun. Different to Fairhall and Caramazza (2013) and Devereux et al. (2013), participants were not required to implicitly or explicitly reference the superordinate object category and different to Reddy et al. (2010) and Lee et al. (2012) participants were not asked to rehearse vivid mental imagery of the stimuli prior to the experiment or to specifically visualize the object.

\section{fMRI acquisition and preprocessing}

Just et al. (2010) recorded functional images on a Siemens Allegra 3.0 $\mathrm{T}$ scanner, with a gradient echo EPI pulse sequence $(\mathrm{TR}=$ $1000 \mathrm{~ms}, \mathrm{TE}=30 \mathrm{~ms}$ and $60^{\circ}$ degree flip angle). Seventeen $5 \mathrm{~mm}$ thick oblique-axial slices were imaged with a $1 \mathrm{~mm}$ gap between slices
NEAR

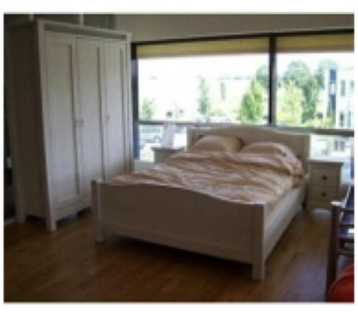

FAR

bed

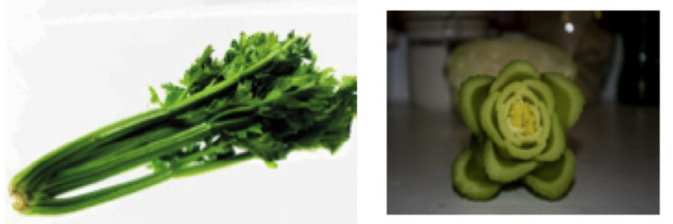

desk
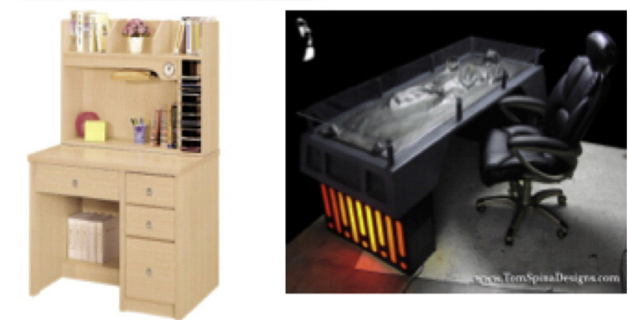

$\operatorname{dog}$
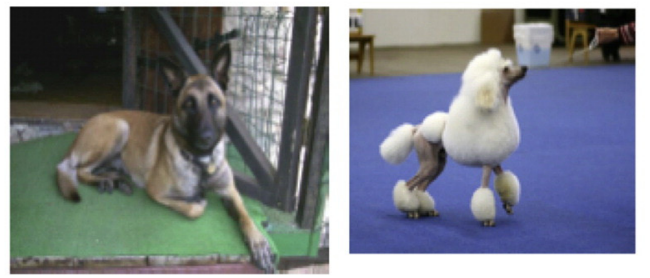

Fig. 3. Example pictures whose BoVF representations are nearest to (left) viz. farthest from (right) the average vector representing the corresponding concept.

and the acquisition matrix was $64^{*} 64$ with $3.125 \mathrm{~mm}^{*} 3.125 \mathrm{~mm}^{*} 5 \mathrm{~mm} 279$ voxels. They subsequently corrected data for slice timing, participant 280 motion and linear trend, before normalizing to MNI space and resam- 281 pling to a $3 * 3 * 6 \mathrm{~mm}^{3}$ grid. The voxel-wise percentage signal change be- 282 tween stimulus and fixation conditions was calculated, and the mean of 283 the 4 images acquired $4 \mathrm{~s}$ after stimulus onset was taken to represent 284 each word. Voxel values within each word were normalized by 285 subtracting the mean and dividing by the standard deviation. To create 286 a single brain representation per word per participant, we took the 287 mean per voxel of the six presentations of each word. To create a single 288 category representation per participant, we took the mean per voxel of 289 all presentations of all words in that category.

Table 1

Classes and words from Just et al. (2010) covered by ImageNet.

$\mathrm{t} 1.2$

\begin{tabular}{ll}
\hline Animals & Bear, Cat, Cow, Dog Horse \\
Buildings & Apartment, Barn, Church, House \\
Building parts & Arch, Chimney, Closet, Door, Window \\
Clothing & Coat, Dress, Pants, Shirt, Skirt \\
Furniture & Bed, Chair, Desk, Dresser, Table \\
Insects & Ant, Bee, Beetle, Butterfly, Fly \\
Kitchen utensils & Bottle, Cup, Glass, Knife, Spoon \\
Man-made objects & Bell, Key, Refrigerator, Telephone, Watch \\
Tools & Chisel, Hammer, Screwdriver \\
Vegetables & Celery, Corn, Lettuce, Tomato \\
Vehicles & Airplane, Bicycle, Car, Train, Truck \\
\hline
\end{tabular}




\section{Computational image-based semantic models}

We describe here the procedure we adopted to construct our imagebased representations of concepts. First we extract low-level visual descriptors from a large image collection, inducing a higher-level vocabulary of discrete features capturing simple visual properties that, following standard usage in the computer vision literature are called with the slightly misleading term "visual words". To avoid confusion here between "visual words" and the visually presented written word experimental stimuli, we will use the term "visual features" in place of "visual words". We represent the contents of a single image with a vector recording how many times each visual feature occurs in it. This is the so-called Bag-of-Visual-Words representation of the image (Csurka et al., 2004; Sivic and Zisserman, 2003), which we here shall refer to as Bag-ofVisual-Features (BoVF). Following up on our recent work (Bruni et al., 2014), we approximate the visual meaning of a concept (as opposed to a single depiction of it) by averaging the BoVF vectors of all images that are captioned with the relevant concept name in a large image corpus. The pipeline to extract concept representations from sets of images is summarized in Fig. 1. The model construction parameters described in this section were picked without tuning, based on their effectiveness in our earlier experiments.

\section{Source data}

We retrieved pictures from ImageNet (Deng et al., 2009), one of the largest freely available image databases (14 M pictures), with each picture manually captioned. We chose ImageNet for its high-quality images and labels, and because it provides bounding boxes localizing the object within a picture. Despite its size, ImageNet does not contain pictures with bounding boxes for 9 concepts used by Just et al. (2010), namely arm, carrot, eye, foot, hand, igloo, leg, pliers, saw (in Just et al., 2010), so we run our experiments on 51 concepts with a total of 17,765 images (350 images per concept on average).

\section{Low-level feature vector extraction}

Scale Invariant Feature Transform (SIFT) is one of the most effective low-level descriptor extraction methods for computer vision tasks such as object recognition and image retrieval (Grauman and Leibe, 2011; Lowe, 2004). Its success is due to its robustness to image scale, noise, distortion and partial invariance to illumination changes. SIFT produces descriptors that are actually 128-dimensional vectors encoding the magnitude gradients (directional changes in intensity) within a given image patch. In our implementation, we extract SIFT vectors from the whole image at regular pixel intervals. In particular, we densely sample the image on a regular grid, iterating through the image in steps of 5 pixels, at 4 different scales $(10,15,20,25$ pixel radii), zeroing descriptors that correspond to very low contrast regions (which results in a small portion of the selected pixels not being associated to SIFT vectors). SIFT features are extracted for each component of the HSV (Hue, Saturation and Value) color space, resulting in $3 \times 128-$ dimensional vectors, with 128 features per channel. Thus, in general, from an image with $\mathrm{N}$ pixels, we extract in the order of N/5 SIFT vectors.

Visual feature dictionary construction and bag-of-visual-features representation of specific images

We construct a visual dictionary by clustering all SIFT vectors extracted from our dataset into $25 \mathrm{~K}$ clusters (visual features), using a Gaussian mixture model (GMM), which can be seen as a probabilistic visual vocabulary that approximates the SIFT vector distribution via softclustering (Chatfield et al., 2011). To take uncertainty into account, each SIFT vector in the collection is associated to a probability distribution over clusters (visual features), instead of being assigned to the single nearest centroid. We use a large visual feature dictionary to account for the wealth of visual information encountered in the $18 \mathrm{~K}$ images 350 from our collection. We will later reduce the full feature space to a 351 small set of more general features, sufficient to represent the 51 con- 352 cepts of interest, as described below (Visual concept representations 353 paragraph).

For the representation of a specific image, each of its SIFT vectors is 355 matched to the dictionary, and counted as an instance of the visual fea- 356 ture with the nearest centroid. For this last operation we use Fisher 357 encoding, which captures average first and second order (mean and co- 358 variance) differences between GMM centers and SIFT vectors. The 359 image as a whole is then represented in "visual feature space" by a 360 higher-level vector that records how many times each visual feature oc- 361 curs in it.

The original BoVF method completely discards information about 363 the relative location of visual features in the image. In our implementa- 364 tion, we preserve partial spatial information by dividing the image into 365 8 regions, repeating the BoVF pipeline for each region and concatenat- 366 ing the resulting vectors (Grauman and Darrell, 2005; Lazebnik et al., 367 2006). The final vectors thus contain $200 \mathrm{~K}$ dimensions ( $25 \mathrm{~K}$ visual 368 features $\times 8$ regions). While the choice of preserving just approximate 369 spatial information might be surprising, it insures that the BoVF ap- 370 proach is robust to the infinity of possible variations in part locations. 371 The head of an animal will generally be above its legs, but a model 372 attempting to encode precise head location would fail to generalize 373 across pictures in which the head is on one or other side, from far or 374 from near, etc. (Grauman and Leibe, 2011). Leeds et al. (2013) have ob- 375 served that BoVF representations of images correlate with neural repre- 376 sentations in the ventral visual pathway, suggesting that spatial 377 information might not play such a crucial role as intuitively expected 378 in human object recognition either.

To give intuition about the information that visual features are cap- 380 turing, we utilize the backprojection technique of Yanulevskaya et al. 381 (2012), allowing us to highlight all the pixels in an image whose SIFT 382 vectors were assigned to a specific visual feature. Figs. 2a-e show all 383 the pixels associated to two frequent visual features (with arbitrary yel- 384 low and green color coding) in a few pictures. "Yellow" visual features 385 cluster on horizontal lines, "green" ones along somewhat oblique 386 curvy lines. Consequently, the first visual feature is discriminative of 387 buildings (Figs. 2a,b) and the second of animals (Figs. 2c,d), but there 388 are exceptions, such as the building in Fig. 2e, which features both 389 shapes, and consequently many instances of both visual features. As 390 these examples illustrate, it is not possible to directly map visual fea- 391 tures to any obvious high-level semantic denotation (e.g., object 392 parts). However, image patches linked to the same visual feature cap- 393 ture basic but interpretable visual properties, such as simple shapes. 394

Visual concept representations

Following our previous work (Bruni et al., 2014), we derive a BoVF 396 vector representation of a concept (e.g., a dog) by summing the (nor- 397 malized) BoVF vectors of all images in our collection that are labeled 398 with the target concept (e.g., all dog pictures). We then apply two trans- 399 formations to the resulting aggregated count vector. First, raw counts 400 are transformed into non-negative Pointwise Mutual Information 401 (PMI) scores (Church and Hanks, 1990), assigning larger weights to vi- 402 sual dimensions that are more discriminative across concepts. Finally, 403 we apply the Singular Value Decomposition technique (Manning et al., 404 2008) to the concept vectors, reducing them to 50 dimensions that gen- 405 eralize across the original BoVF features, with almost no loss of variance 406 in the representation of the 51 concepts of interest, but a much more 407 compact encoding of the relevant information.

The resulting "average" concept vectors abstract away from more id- 409 iosyncratic features of specific images, and capture their commonalities, 410 thus approximating a data-induced prototype representation of the vi- 411 sual aspects of a concept. Fig. 3 illustrates how our aggregated vectors 412 naturally encode prototypical representations. We present there, side 413 
by side, a specific picture whose vector is very near the average for the corresponding concept and a picture (still labeled with the relevant concept) that is quite far from the average vector (note that both kinds of pictures were used to construct the aggregated concept vectors). Clearly, pictures near the concept vector depict instances that are more prototypical than those that are far from it.

Image-based vectors were constructed with VSEM, an open library for visual semantics (Bruni et al., 2013; http://clic.cimec.unitn.it/vsem/).

\section{Computational text-based semantic models}

A long tradition of studies in computational linguistics and cognitive science has shown that it is possible to extract empirically effective representations of word meaning by using other words (or other linguistic units) that tend to naturally co-occur with a target term as semantic features a vector-based representation of its meaning (the resulting representations are often called distributional semantic models; see, e.g., Clark, 2013; Landauer and Dumais, 1997; Lenci, 2008; Lund and Burgess, 1996; Turney and Pantel, 2010). Again, the parameters of our linguistic vectors were picked without tuning, based on their effectiveness in our earlier work (Bruni et al., 2014).

We record co-occurrences with collocates within a window of a fixed size of 2 to left and right of each target word. Co-occurrence statistics are gathered from the freely available ukWaC and Wackypedia corpora combined, containing about 3 billion words in total (http://wacky. sslmit.unibo.it/). As collocates, we select a subset of $30 \mathrm{~K}$ words, composed by the top $20 \mathrm{~K}$ most frequent nouns, $5 \mathrm{~K}$ most frequent adjectives and $5 \mathrm{~K}$ most frequent verbs. Similar to the image-based vectors, we reweight and reduce the dimensionality of text vectors. However, unlike the approximately normally distributed visual feature counts, word frequencies in word corpora are heavily skewed with a prevalence of very rare types (the so-called "Zipfian" distribution). Since PMI is known to severely overestimate the importance of rare types, we correct non-negative PMI scores multiplying them by the corresponding raw co-occurrence counts (Evert, 2005; Manning and Schütze, 1999). As with the visual vectors, we then apply Singular Value Decomposition down to 50 dimensions.

\section{Representational similarity analysis: general procedure and terminology}

RSA compares the way the same referents are organized in different representational spaces (such as activation patterns in different areas of the brain and feature-based computational representations). We might expect a snake to occupy a similar position to a belt in visual space, but be far separated in linguistic semantic space, and RSA follows this intuition by systematically comparing paired item similarities. In the resulting common pairwise similarity space, we anticipate that visual similarity structuring (image-based models and visual brain areas) would be differentiable from linguistic structuring (text-based models and linguistic brain areas). In other words, that the matrix of paired similarities derived from a brain region of interest (ROI) that is visually specialized should correlate more with the matching matrix derived from image-based models (Im) than text-based models (Tx), and vice-versa.

The abstraction to similarity space allows multiple participants' brain data to be easily combined in an average similarity matrix, thus capturing group-level commonalities (and side stepping some problems surrounding imprecisions in spatial normalization of crossparticipant data to the same anatomical space - in our case all participants were normalized to MNI space). This is beneficial if there are group-level commonalities in representational similarity (as opposed to individual representational schemes), in which case averaging across participants will bring out these regularities and cancel out noise in individual participants' data. It is also relevant that our computational semantic models are at group level, built from photographs taken by many people and text written by many authors. Although individual- level computational models are a theoretical possibility (e.g., built 476 from an individual's documents and photography), their creation was 477 infeasible for the current study.

We conduct hypotheses tests at both group-level (on mean similar- 479 ity matrices) and individual-level (where hypotheses are first tested on 480 individual's similarity matrices and then in a second-level analysis a one 481 sample t-test is used to test whether the resultant set of individual-level 482 test statistics differs from zero). Whilst group-level analyses are more 483 likely to detect a pattern in the data (because the effects of noise are re- 484 duced), as a reviewer pointed out, we are still treating participants as 485 fixed, rather than random effects, meaning that the inferences we 486 draw might not generalize to other individuals from the population. 487 We leave a more complex mixed-effects analysis to further work. We 488 summarize results of both group and individual hypothesis tests in the 489 main article and list all results in detail in Supplementary Materials. 490 The more succinctly reportable group-level results covers all experi- 491 mental and exploratory ROIs in both hemispheres (as defined in the 492 next section). Individual-level results, which lead to the same conclu- 493 sions, are provided for the key experimental regions (because results 494 are lengthier to report, tabulation in detail is in Supplementary Mate- 495 rials 6).

Specifically, for each computational model or ROI, the representa- 497 tional similarity structure (denoted by ss) was estimated by taking 498 Pearson's correlation coefficient between all 1275 unique word pair 499 combinations, given by the lower/upper off diagonal triangle of the 500 51*51 symmetric paired similarity matrix (e.g., $\operatorname{Im}_{s S}$ is the list of 1275501 image-based paired similarities). Group-level similarity structures for 502 each ROI were obtained by taking item-wise mean similarities. $\quad 503$

The similarity structure of models and ROIs was compared using 504 Spearman's correlation $(\mathrm{n}=1275)$. Statistical significance was evaluat- 505 ed using a permutation test as described in Kriegeskorte et al. (2008a): 506 The word labels of one of the two pairwise similarities matrices under 507 comparison were randomly shuffled, rows and columns reordered ac- 508 cordingly, and the resulting similarity structure correlated with the 509 other. This process was repeated 10,000 times and the p-value taken 510 as the proportion of times the permuted correlation coefficient was 511 greater than or equal to the observed correlation coefficient. 512

We repeated analyses at class-level (with classes defined by the 513 original data set) following the reasoning that averaging representa- 514 tions within classes should let more general patterns emerge in the in- 515 herently noisy fMRI data. As the class man-made objects is in essence a 516 superordinate category, that could reasonably subsume other test clas- 517 ses such as tool and kitchen utensils, it was left out of the class-level anal- 518 yses. In the class-level RSA, there were 10 classes and 45 unique class 519 pairs (RSA results including the left-out class are documented in Supple- 520 mentary Materials Figure S2 and Table S2 and do not change interpreta- 521 tion). To create a group class-level similarity structure for each ROI, the 522 item-wise mean similarity structure was taken across participants (45 523 mean similarities for 10 classes).

\section{Hypotheses of visual/linguistic semantic representational} dominance in different brain regions

Hypotheses predicting whether image- vs. text-based similarity 527 structure would correlate more with specific ROIs were predominantly 528 inspired by recent multivariate fMRI analyses of object representation, 529 in particular those using RSA.

\section{Visual dominance}

Object/shape mental imagery can be discriminated in the ventral 532 temporal cortex and lateral occipital areas and decoding of imagery is 533 possible using classifiers trained on perceptual data (Lee et al., 2012; 534 Reddy et al., 2010; Stokes et al., 2009a, 2009b; Stokes et al., 2011). Q6 This, on top of a number of multivariate analyses demonstrating object 536 classification in the ventral-temporal stream when participants were 537 
cued by images (Connolly et al., 2012; Fairhall and Caramazza, 2013; Haxby et al., 2001), and Leeds et al.s' (2013) RSA correlating fMRI activation in the ventral-temporal-cortex cued by photographs corresponding to the words we analyze and SIFT-based models of the image stimuli lead to the hypothesis that image-based models would show significantly greater correlation with ventral temporal and lateral occipital areas.

\section{Linguistic dominance}

Our starting point for predicting linguistically dominant ROIs was Fairhall and Caramazza (2013) and Devereux et al. (2013), who used RSA to compare fMRI representations of five/six object categories, elicited by both pictures and words to semantic models, as both stimulus modalities should cue linguistic representations. Fairhall and Caramazza (2013) found that the left posterior-mid/inferior-temporal gyrus and posterior-cingulate/precuneus both support cross-modal classification and reflect the category structure of models derived from WordNet. Echoing this, Devereux et al (2013) found semantic category structure elicited by both text and pictures in the left middle temporal gyrus and left posterior cingulate/precuneus, and in addition in the right posterior cingulate/precuneus, inferior parietal lobe, left inferior frontal gyrus (in particular pars triangularis), left/right precentral gyrus and right superior frontal regions. Only representations in the intra-parietal-sulcus maintained a semantic category structure that did not change in response to text or image presentation, however pictures could elicit different or more specific linguistic-semantic representations than words, see also our Discussion, and Glaser (1992) for extensive coverage of behavioral experiments comparing picture/ word stimuli in cognitive tasks.

As reading text may evoke visual simulations, viewing images may evoke linguistic representations, and image and text-based semantics may correlate (e.g., in context/situation), decoding brain activity elicited by different stimulus modalities across modalities is no guarantee of a strictly cross/amodal brain representation. However as the above regions were distinct from the regions we hypothesized to be visually dominant we provisionally considered them as candidates for linguistic knowledge representation.

We next referenced these regions to a recent review of semantic memory (Binder and Desai, 2011; Binder et al., 2009). The left midtemporal gyrus and left posterior inferior parietal lobe have an established role in entity and event knowledge representation, whilst the left inferior frontal gyrus is implicated in knowledge evaluation/selection/retrieval and as such we might expect to detect specific aspects of semantic representation filtering/filtered from a general knowledge base stored elsewhere. We assigned all three regions hypotheses of text dominance. The posterior cingulate/precuneus is comparatively less well understood, with a possible role in episodic memory retrieval, irrespective of the memory's imagery content (Krause et al., 1999) and with stronger ties to encoding scene familiarity (real vs fictitious) as opposed to scene reconstruction (Hassabis et al., 2007). As both Fairhall and Caramazza (2013) and Devereux et al. (2013) detected semantic category structure here (possibly related to context/situation), we also assigned the posterior cingulate/precuneus a hypothesis of text dominance. The role of the superior medial frontal cortex is somewhat obscure (possibly translating affective states to a coordinated plan for knowledge retrieval) and therefore we analyzed it without a prediction.

\section{Equivalent image/text correlation}

Strong evidence that object representations in the inferior temporal gyrus interface visual and linguistic semantic knowledge is provided by Kriegeskorte et al. (2008b) and Carlson et al. (2014). The first analysis successfully correlated human fMRI data elicited by 92 color photographs of faces and bodies of animals and humans, and of natural and artificial objects to primate brain data. The second analysis demonstrated that the similarity structure of the same data correlates 600 with text-based semantic models similar to those we use. We therefore 601 predicted that both image- and text-based models would correlate with 602 the left-inferior-temporal gyrus.

\section{Exploratory analysis}

Some ROIs were analyzed because of their posited roles in process- 605 ing semantic knowledge (Binder and Desai, 2011; Binder et al., 2009), Q10 but without a dominance hypothesis, either because of their association 607 with other modalities and/or their connection to our models is margin- 608 al. These were the precentral and supramarginal gyrii (motor/action), 609 superior temporal and Heschl's gyrii (audition) and the dorsomedial 610 frontal cortex.

\section{Voxel selection in experimental ROIs}

ROIs were partitioned using Tzourio-Mazoyer et al.s' (2002) auto- 613 matic anatomical labeling (AAL) scheme. Individual AAL regions were 614 combined as below (see also Fig. 4) into a total of eleven left hemispher- 615 ic (L) and right hemispheric (R) sets. Hypotheses were defined to apply 616 primarily to the left hemisphere, in line with the common observation 617 of (right handers') left hemispheric dominance in semantic tasks 618 (e.g., Binder et al., 2009) and mental imagery (e.g., Ishai et al., 2000), 619 and Just et al.s' (2010) observation of left dominant activation on our 620 data set. The hypotheses were reflected to the right hemisphere 621 expecting weaker correlations.

622

As there was no strong a priori reason to predefine the number of 623 voxels to analyze we repeated analysis within each ROI on the 624 $\{50,100,200$ and 400$\}$ most stable voxels: Pearson's correlation of each 625 voxel's activity between matched words in all scanning session pairs 626 (15 unique session pairs giving 15 correlation coefficients of 51627 words) was computed and the mean coefficient used as stability mea- 628 sure (as per Mitchell et al., 2008). Experimental hypotheses were the 629 same irrespective of number of voxels. If there were not 400 voxels in 630 the ROI, the entire ROI was analyzed (number of voxels per ROI per par- 631 ticipant are in Table S9).

AAL templates were as follows (defined for left hemisphere here, but 633 combinations repeated for right). Image dominance: (Fig. 4-Blue): 634 LMOG $=\{$ Occipital_Mid_L, LVTC $=\{$ Fusiform_L \& ParaHippocampal_L $\} .635$ Text dominance (Fig. 4-Yellow): LMTG $=$ Temporal_Mid_L; LPIP $=636$ \{Angular_L, Parietal_Inf_L\}; LIFG \{Frontal_Inf_Tri_L, Frontal_Inf_Oper_L\}; 637 $\mathrm{LPCP}=\{$ Precuneus_L, Cingulum_Post_L $\} ;$ Equivalent image/text correla- 638 tion (Fig. 4-Green): LITG = Temporal_Inf_L; Exploratory analysis: (Fig. 4- 639 Red): $\mathrm{LPG}=$ Precentral_L; $\mathrm{LSMG}=\{$ Supramarginal_L $\} ;$ LSTG $=640$ \{Heschl_L, Temporal_Sup_L\}; LDMFC $=$ \{Frontal_Sup_Medial_L, 641 Frontal_Sup_L\}.

\section{Results}

Hypothesis-driven analysis: Image-based models detect embodied repre- 644 sentations in visual areas

As set out in the introduction, to positively identify a visually embod- 646 ied representation, the image-based model should show both a signifi- 647 cantly stronger correlation than the text-based model in visual regions 648 and weaker correlation in hypothesized non-visual regions (even if 649 both models correlate with the same region, which is not unexpected 650 given that the stimuli were not picked to contrast visual and linguistic 651 similarity, and that visual/linguistic referents may occur in similar con- 652 texts). As $I m_{s s}$ and $T x_{s s}$ were significantly correlated (word-level, $\rho=653$ $.39, \mathrm{p}<.001, \mathrm{n}=1275$, class-level $\rho=.42, \mathrm{p}<.001, \mathrm{n}=45$ ), we 654 employed Steiger's (1980) test of difference between dependent corre- 655 lations using the $\mathrm{T}_{2}$ statistic.

The hypotheses were tested at both word and class levels. Pooling 657 results for each lateralized anatomical ROI there were 8 tests of image/ 658 


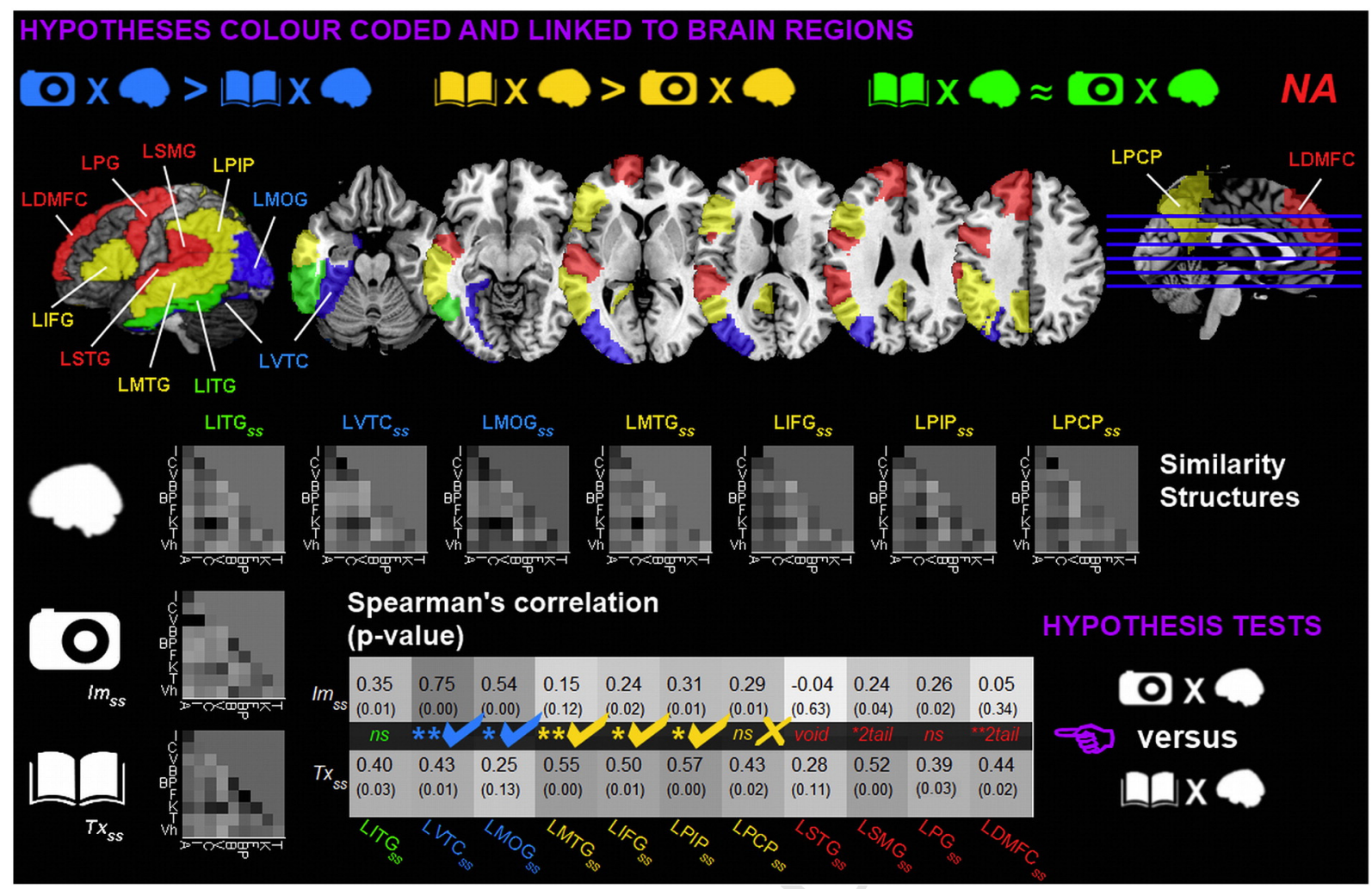

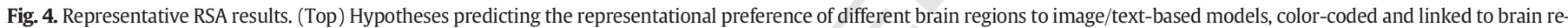

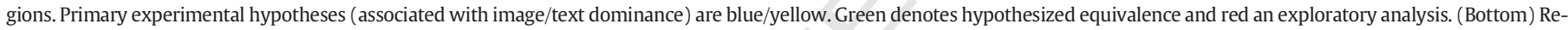

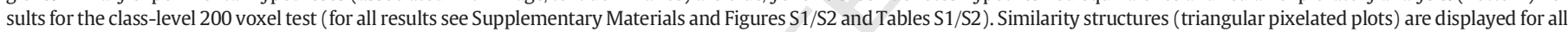

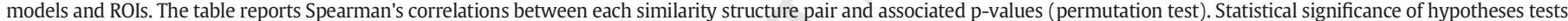

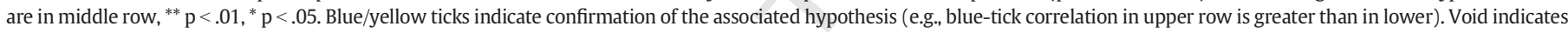
that neither image/text-based model correlated significantly with the ROI.

text modality preference (word/class-level $*\{50,100,200,400\}$ voxels). If neither computational model significantly correlated with the ROI, the difference test was declared void (9/96 tests). The number of hypotheses supported in non-void tests at group level was as follows (all grouplevel tests and a word length control analysis is documented in Supplementary Materials 1 Figures S1/S2 and Tables S1-S3): LMOG (8/8), RMOG (5/8), LVTC (8/8), RVTC (3/8), LMTG (7/7), RMTG (4/4), LIFG (6/8), RIFG (7/7), LPIP (5/8), RPIP (6/6), LPCP (1/7) \& RPCP (3/8). Correction for false discovery rate $(\mathrm{q}=.05)$, conducted separately at word-level and class-level, leads to the following amendments RVTC (0/8), LIFG (5/8), LPIP (4/8), LPCP (1/7). Zero tests were contradicted. Significant correlations between LITG and both models were present in all but two tests and there were no significant differences in correlation strength between models (as anticipated). RITG however was found only to correlate with the text-based model at class-level in two tests, where the correlation was significantly greater than with the image-based model.

Of the exploratory ROIs tested, only LSMG showed a significant word-level correlation (with both $I m_{s s}$ and $T x_{s S}$ ). At class-level, we found significant correlations of all exploratory ROIs with $T x_{s s}$. Significant differences in the strength of correlations between $\operatorname{Im}_{s S}$ and $T x_{s s}$ were observed, especially in the right hemisphere where correlations with $I m_{s S}$ were around zero.

Representative RSA results comparing $I m_{s s}$ and $T x_{s s}$ correlations with ROIs at class-level (200 voxels) are in Fig. 4. To support that these results are representative we verified that the overall result pattern were consistent across hemispheres and when tested with different numbers of stable voxels. Specifically, lists of correlation coefficients of $I m_{s s}$ and $T x_{s s}$ with each $\mathrm{LROI}_{s s}$ were stacked $(\mathrm{n}=22)$, the same stacking repeated for the right hemisphere, and the two resulting hemispheric lists were compared using Spearman's rank correlation at each test scale
$(\{50,100,200,400\}$ voxels). The correlations are uniformly high (word- 690 level $\rho=\{.71, .84, .82, .77\}$, all $\mathrm{p} \leq .001$; class-level, $\rho=\{.65, .75, .81, .77\} 691$ all $\mathrm{p} \leq .002)$. As anticipated, correlations were significantly greater in 692 the left hemisphere, where the difference between the above data sets 693 was tested using Wilcoxon signed rank tests: At word-level $\mathrm{W}=694$ $\{15,8,21,38\}$ all $p \leq .004$. At class-level $W=\{51,52,39,42\}$ all $p \leq .015$. Fi- 695 nally and not surprisingly word and class-level results at each scale 696 were strongly related, as tested by correlating all word-level correla- 697 tions pooled across hemispheres with all pooled class-level results, giv- 698 ing $\rho=\{.82, .79, .82, .82\}$, all $\mathrm{p} \leq .001, \mathrm{n}=44$.

The number of hypotheses supported in individual-level tests that 700 focused on the key left-hemispheric experimental ROIs were LMOG 701 (6/8), LVTC (5/8), LMTG (3/8), LIFG (8/8), LPIP (5,8), LPCP (1/8). There 702 were no tests declared void and no contradictions. Tests that were not 703 passed for LMOG, LVTC and LPIP were all at class-level. This is likely at- 704 tributable to lower analytic power with fewer classes and less data (as 705 the class man-made objects was excluded from the class-level analysis). 706 All individual-level results are comprehensively documented in Supple- 707 mentary Materials 6, Tables S10-S15.

As a further check, we ran a second set of analyses explicitly testing 709 the sensitivity of ROIs to both models, to check for cases where one 710 model has stronger correlation than the other but the weaker model 711 still significantly explains variance in that ROI. We regressed ROIss on 712 one model's similarity structure and tested whether the residuals corre- 713 lated with that of the other model. This was then repeated exchanging 714 the models. Prior to regression, correlation coefficients in all similarity 715 structures were hyperbolic-arc-tangent transformed according to Fish- 716 er's $r$ to $z$ transformation. These secondary analyses were run on the 717 key left hemispheric ROIs that had been assigned modal dominance hy- 718 potheses, which were translated as follows. For an ROI hypothesized to 719 be visually dominant the residuals following regression on the text- 720 
based model were predicted to positively correlate with the imagebased model. When regression is on the image-based model, no positive correlation between the resultant residuals and the text-based model was predicted. Image and text-based models were switched accordingly for ROIs hypothesized to be linguistically dominant.

These analyses yielded similar results, with the comparative number of predictions supported being LMOG (16/16), LVTC (15/16), LMTG (14/ 14), LIFG (16/16), LPIP (13/16), LPCP (16/16), (there are double the number of tests comparative to the previous analysis, because rather than directly comparing image and text-correlations, we test first for visual sensitivity once text-based semantic effects are regressed out, and second for linguistic sensitivity once image-based effects are regressed out). In LVTC, at class-level, there was a single instance of significant correlation with the text-based model following removal of the image-based-model's regression line, and for LPIP one instance of this, and two of the counter-case of significant correlation with the imagebased model after removal of the text trend at word-level. Detailed results are in Supplementary Materials 1 (Tables S3 and S4). Individuallevel tests returned a similar outcome with the number of supported predictions being: LMOG (15/16), LVTC (14/16), LMTG (16/16), LIFG (16/16), LPIP (14/16), LPCP (13/16). Full documentation is in Supplementary Materials 6 (Tables S16-S19).

In summary hypothesis tests of the relative correlation strengths between $I m_{s s}$ and $T x_{s s}$ and different ROIs favorably matched expectation. ROIs predicted to show visual dominance (L/RMOG, L/RVTC) did so in the vast majority of tests, and L/RMTG, L/RPIP and L/RIFG showed text-dominance also in line with prediction. We consider this as strong evidence that visually embodied object representations are activated as people read and contemplate object words, without visual object stimulation. L/RPCP were difficult to interpret (detailed results are in Supplementary Materials 1 Figures S1/S2 and Tables S1/S2) and we provisionally consider these ROIs to be weakly defined as regards our hypotheses.

\section{Distribution of local image/text-based correlations throughout the brain}

Searchlight RSA (Connolly et al., 2012; Devereux et al., 2013; Fairhall and Caramazza, 2013; Kriegeskorte et al., 2006; Leeds et al., 2013) were run to visualize the spread and relationship between localized image/ text correlations throughout the brain. Searchlight was run at word and class-level. For space reasons, and as results are similar, class-level results are in Supplementary Materials 2. Firstly the set of all grey matter voxels in the brain that were common to all participants was identified by intersecting the MNI normalized grey matter voxel masks across participants. A contiguous voxel 'sphere' with a radius of $9 \mathrm{~mm}$ (mean \pm std voxels: $56.5 \pm 12.7$ ) was iteratively centered on each voxel of the common MNI mask of all grey-matter voxels. At each sphere location, neural similarity structures $\left(\mathrm{SROI}_{s S}\right)$ were created individually for each participant in exactly the same way as the previous ROI analysis, but based only on voxels in the searchlight sphere. In order to capitalize on group level regularities, each $\mathrm{SROI}_{s S}$ was averaged across all participants at each location. The mean $\mathrm{SROI}_{s S}$ at each location was then correlated with $I m_{s s}$ and $T x_{s s}$ using Spearman's $\rho$.

A permutation test was used to compute the p-value of the correlation coefficient at each sphere location. Following Leeds et al. (2013), word labels were permuted 500 times. The mean and variance of the correlation between the resultant shuffled similarity structures and the actual brain $\mathrm{SROI}_{s S}$ were used to transform correlation coefficients to $\mathrm{z}$-scores. A one-tailed $\mathrm{p}$-value was then obtained using $\mathrm{p}=1$ $\operatorname{erf}(z)$ where erf is the cumulative density function of a standard normal distribution. Similarity maps of $I m_{s s}$ and $T x_{s s}$ were thresholded by false discovery rate $(\mathrm{q}<.1)$ (Genovese et al., 2002). Significant $\rho^{\prime} \mathrm{s}(\mathrm{p}<.01$ from FDR) were plotted in MNI space.

Fig. 5 displays word-level searchlight results, correlation clusters are in Tables 2 and 3 for the image and text-based models respectively (class-level results are in Figure S3, Tables S6 and S7). Cyan/Orange indicates localized regions significantly correlated with $I m_{S S} / T x_{S S}$ respec- 785 tively. Brown indicates overlap in significant correlations. Significant 786 correlations with $I m_{s S}$ only were largely located in the bilateral occipital 787 cortex, the precuneus and in ventral regions of the temporal cortex 788 (with slight left hemispheric bias) in line with the general visual domi- 789 nance previously confirmed in L/RMOG and L/RVTC. Significant correla- 790 tions with $I m_{s s}$ were also detected within LIFG (particularly pars 791 opercularis) and LSMG. The previous hypothesis testing analysis con- 792 firmed LIFG as linguistically-dominant despite significant correlation 793 with $I m_{s s}$. LIFG might be selectively filtering aspects of broader semantic 794 representations and intermittently processing visual and non-visual in- 795 formation: in this case, the image-correlated representations observed 796 are unlikely to be directly related to visual processing. $\quad 797$

Significant correlations with $I m_{s s}$ were more widespread than $T x_{s s} .798$ One interpretation is that visual simulations have more widespread ac- 799 tivity traces than linguistic representations (which would be consistent 800 with Glaser, 1992, who observed that pictures produce stronger prim- 801 ing effects than words). However the result is also on face value at 802 odds with the hypothesis-driven ROI analyses (where more ROIs 803 showed linguistic dominance). The difference is attributable to the 804 two analyses operating at different spatial scales (the searchlight 805 looking at all voxels within a small sphere comparative to the ROI anal- 806 yses which selects stable voxels from a larger volume). As we don't 807 know what spatial scale(s) visual simulations and linguistic cognition 808 operate upon, and how visual/linguistic modalities interact, we remain 809 agnostic on the interpretation of this result.

Significant correlations with $T x_{s s}$ were observed in similar/neighbor- 811 ing regions to $I m_{s s}$ and infrequently detected in the right hemisphere, 812 consistent with the standard finding of left hemispheric dominance in 813 language tasks. Isolated correlations were observed in LPIP (including 814 the angular gyrus), L/RVTC, L/RPCP, with smaller patches in LIFG and 815 LITG/LMTG. The spread was consistent with the hypothesis testing anal- 816 ysis (and the comparative sparseness is a byproduct of the focal nature 817 of searchlight analysis as identified above).

Areas of overlap between $I m_{S S}$ and $T x_{S S}$ correlation, suggestive of a 819 transition between visual aspects of object representations and 820 higher-level linguistic semantics, were found in LITG (consistent with 821 our expectation of high-level visual object representations in this re- 822 gion), LPIP, L/RPCP, portions of L/RVTC and within LIFG. Close inspection 823 of the searchlight volume reveals that the multimodal overlap in LVTC is 824 on the boundaries of the perirhinal cortex where Bruffaerts et al.s' 825 (2013) observed that semantic similarity reflects fine-grained within- 826 object-category semantic similarity between words, and more generally 827 this network of areas is strikingly compatible with theories considering 828 semantic memory to incorporate a selection of information conver- 829 gence zones (e.g., angular/supramarginal gyri and sections of inferior/ 830 middle temporal and fusiform gyri) and high-level modulatory areas 831 such as left inferior frontal regions (Binder and Desai, 2011; Binder Q13 et al., 2009; Mahon and Caramazza, 2009; Martin, 2007).

Decoding visual/linguistic brain representations using image and text- 834 based models

If we can decode unlabeled embodied brain representations based 836 only on information from the image-based models, we have evidence 837 that the underlying embodied simulation synthesizes distinguishable 838 visual patterns. If this is the case, we should also be able to utilize the 839 complementary information in image/text-based models to decode dif- 840 ferent ROIs in parallel.

We exploit correspondences between model- and brain-activation- 842 defined similarity spaces in order to guess the stimulus concept classes, 843 without the need for manually labeled training data. Specifically, we 844 adapt the algorithm proposed by Raizada and Connolly (2012) for 845 cross-participant decoding to model-based decoding as follows. We 846 are given the matrix of between-class pairwise similarities produced 847 by a model. For the brain data, we have access to mean activation 848 


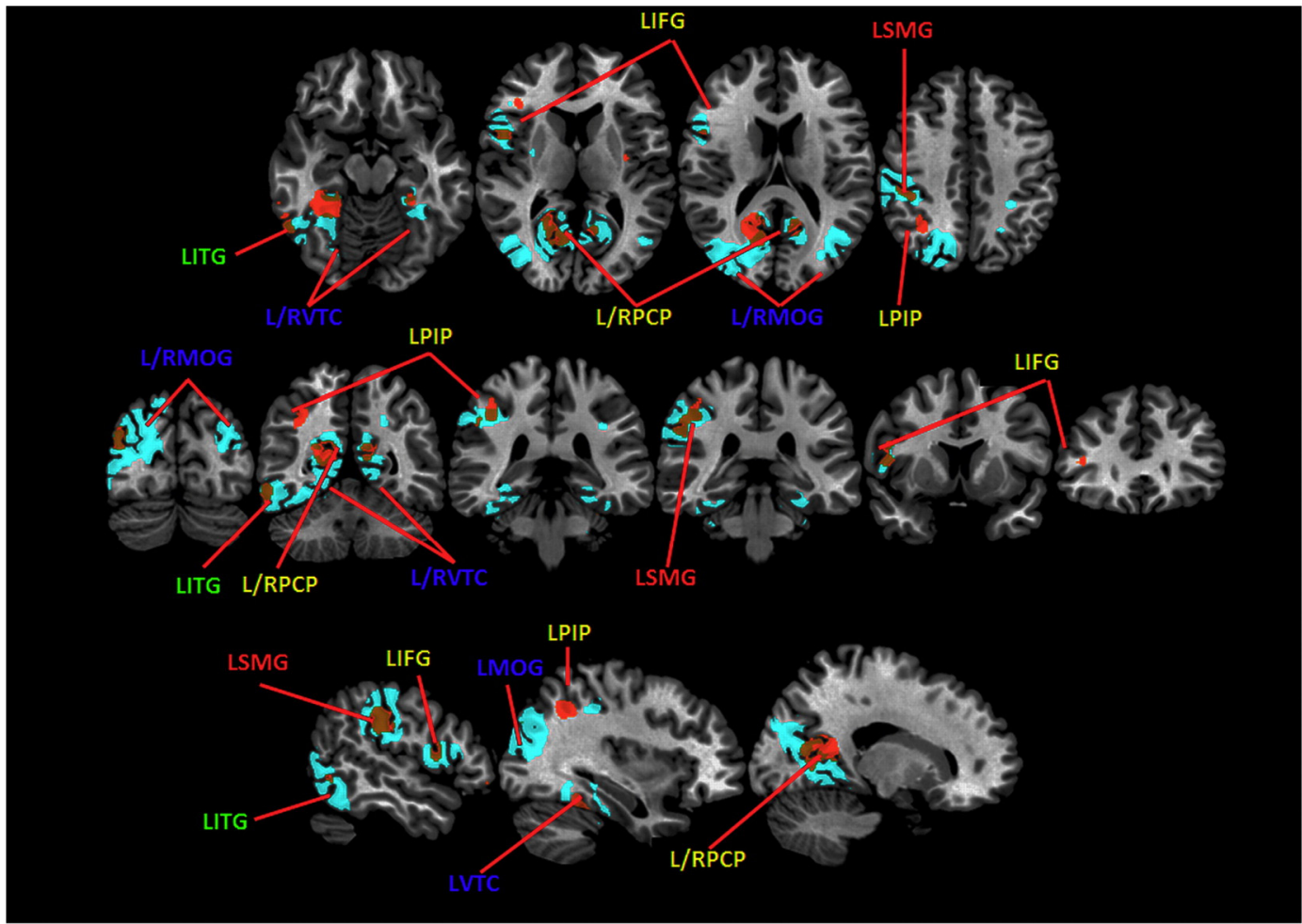

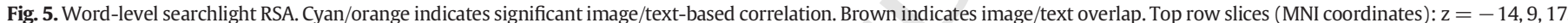
43; mid row: $\mathrm{y}=-79,-56,-37,-33,7,30$; bottom row: $\mathrm{x}=-50,-33,-23$. See Figure S3 for class-level results.

vectors for each class, but not to the corresponding class labels. If the model is accurately capturing the same similarity structure as represented in the brain, we can look for the class label assignments that would make the brain similarity matrix as similar to the model-based matrix as possible. As a toy example, suppose that in model space we measure $\operatorname{sim}($ mammal,insect $)=.70, \operatorname{sim}($ mammal,tool $)=.30$, $\operatorname{sim}($ insect,tool $)=.10$. We are given 3 unlabeled vectors of pooled brain activation data with $\operatorname{similarities} \operatorname{sim}(\mathrm{x}, \mathrm{y})=.83, \operatorname{sim}(\mathrm{x}, \mathrm{z})=.29$, $\operatorname{sim}(y, z)=.11$. Then, it is most reasonable to assume that $x$ corresponds to mammal trials, $y$ to insects and $z$ to tools, since this assignment maximizes second-order similarity between model and brain similarities. More precisely, we exhaustively evaluate all the possible label permutations of the rows and columns of the brain similarity matrix, and pick the one leading to the largest Pearson correlation of the pairwise similarity scores with those in the model-based matrix (in ongoing work we are targeting word-level classification, which, with 51! possible label permutations necessitates approximate methods to search for the best solution).

We use $I m_{s s}$ to decode class labels in LVTC (which showed greatest correlation to $\mathrm{Im}_{s s}$ in the ROI analysis). We repeated decoding on $\{50,100,200,400\}$ voxels. Chance accuracy was $1 / 10$, and equating conventional statistical thresholds to the number of classes correctly decoded (the same threshold applies using either Binomial or permutation testing as per Raizada and Connolly, 2012) gave [3/10 $p<.05]$, [4/10 $\mathrm{p}<.01],[5 / 10 \mathrm{p}<.001],[6 / 10 \mathrm{p}<.0001]$. The number of classes accurately decoded at the four different voxel scales using $I m_{s s}$ was $\{1,2,0,8\}$ and using $T x_{s s}$ was $\{0,0,0,2\}$. For the image-based model only, at 400 voxels, we could successfully decode eight of the ten classes. The instability in decoding at different scales for the image-based model is possibly due to an inadequate representation of all test classes in the 3 lower voxel scales. If visual simulations are naturally more stable for some words rather than others, then voxel selection would have biased towards covering the subset of most stable words. As the decoding algorithm relies on the inter-relationship between all classes 882 this could seriously disrupt decoding accuracy. Given the local text- 883 based correlation observed in the searchlight analysis of LVTC (Fig. 5), 884 it is almost certain that non-visual aspects of semantic information are 885 incorporated in the decoded representation. However, as successful 886 decoding here was only possible based on entirely visual information, 887 and accomplished without training, it is reasonable to assume that the 888 decoded semantic representations are anchored in visual perception. 889 This confirms that internally induced embodied representations contain 890 at least a class-level degree of visual detail for many classes. 891

Nevertheless, the previous effect was brittle, being absent from 3892 of 4 voxel scales. In line with our expectation that linguistic and visu- 893 al model similarities would differentially correlate with brain simi- 894 larities in different regions, we extend the previous procedure to 895 operate by comparing different models with different brain ROIs in 896 parallel. We now assume we have multiple brain region activation 897 vectors for each class (but still don't know their class label). We 898 then select the label assignment that maximizes the (weighted) 899 sum of second-order similarity correlations with the relevant models 900 across regions: That is, we search for a label assignment resulting in 901 both high correlations with the text-based model for regions hy- 902 pothesized to be mostly devoted to linguistic processing and high 903 correlations with the imaged-based model in visual-processing re- 904 gions. This "multimodal" decoding strategy thus capitalizes on 905 targeting more diverse brain representations that are spread across 906 greater brain areas, combining different sources of evidence for 907 more robust decoding.

We determined which computational model to apply to each ROI 909 based directly on our previous 6 modal-specificity hypotheses that 910 linked image/text-based models to different ROIs. This was the first 911 multimodal model configuration we tried. Each potential assignment 912 of class labels to the brain data was scored using the mean weighted 913 correlation in Eq. (1), where $\mathrm{r}(\mathrm{x}, \mathrm{y})$ denotes Pearson's correlation 914 
t2.1 Table 2

t2.2 Image-based model word-level searchlight: Breakdown of AAL regions in significantly cort2.3 related searchlight clusters (sampled at $1 \mathrm{~mm}^{3}$ ) and MNI coordinates of peak correlations 12.4

per ROI (AAL regions identified as per Just et al., 2010).

\begin{tabular}{|c|c|c|c|c|c|}
\hline Cluster & $\begin{array}{l}\text { Vol } \\
\left(\mathrm{mm}^{3}\right)\end{array}$ & ROI & $\mathrm{x}$ & $\mathrm{y}$ & $\mathrm{z}$ \\
\hline \multirow{25}{*}{$\begin{array}{l}\text { Bilateral } \\
\text { Occipital/Temporal/Parietal }\end{array}$} & \multirow[t]{25}{*}{11246} & Occipital_Mid_L & -37 & -80 & 23 \\
\hline & & Calcarine_L & -16 & -62 & 11 \\
\hline & & Lingual_L & -20 & -53 & -5 \\
\hline & & Occipital_Sup_L & -20 & -66 & 23 \\
\hline & & Occipital_Inf_L & -54 & -67 & -11 \\
\hline & & Cuneus_L & -15 & -61 & 22 \\
\hline & & Precuneus_L & -12 & -54 & 16 \\
\hline & & Parietal_Sup_L & -22 & -68 & 44 \\
\hline & & Parietal_Inf_L & -33 & -79 & 42 \\
\hline & & Angular_L & -46 & -76 & 30 \\
\hline & & Temporal_Inf_L & -46 & -52 & -16 \\
\hline & & Fusiform_L & -31 & -44 & -18 \\
\hline & & Temporal_Mid_L & -43 & -72 & 16 \\
\hline & & ParaHippocampal_L & -26 & -37 & -10 \\
\hline & & Cerebelum_6_L & -31 & -45 & -29 \\
\hline & & Cerebelum_4_5_L & -22 & -41 & -25 \\
\hline & & Cerebelum_Crus1_L & -45 & -44 & -31 \\
\hline & & Occipital_Mid_R & 37 & -71 & 28 \\
\hline & & Calcarine_R & 17 & -58 & 11 \\
\hline & & Lingual_R & 14 & -56 & 4 \\
\hline & & Cuneus_R & 15 & -60 & 23 \\
\hline & & Precuneus_R & 12 & -54 & 18 \\
\hline & & Angular_R & 40 & -66 & 22 \\
\hline & & Temporal_Mid_R & 46 & -72 & 21 \\
\hline & & Cerebelum_4_5_R & 10 & -55 & -4 \\
\hline \multirow[t]{4}{*}{ Left Inferior Parietal } & \multirow[t]{4}{*}{2098} & SupraMarginal_L & -53 & -30 & 31 \\
\hline & & Postcentral_L & -52 & -23 & 29 \\
\hline & & Parietal_Inf_L & -50 & -28 & 39 \\
\hline & & Temporal_Sup_L & -58 & -30 & 20 \\
\hline \multirow[t]{4}{*}{ Left Inferior Frontal } & \multirow[t]{4}{*}{569} & Frontal_Inf_Oper_L & -53 & 8 & 11 \\
\hline & & Rolandic_Oper_L & -47 & 3 & 11 \\
\hline & & Frontal_Inf_Tri_L & -58 & 22 & 14 \\
\hline & & Precentral_L & -48 & 6 & 14 \\
\hline \multirow[t]{6}{*}{ Right Ventral Temporal } & \multirow[t]{6}{*}{1684} & Fusiform_R & 32 & -38 & -19 \\
\hline & & Cerebelum_4_5_R & 25 & -38 & -26 \\
\hline & & Cerebelum_6_R & 33 & & -29 \\
\hline & & ParaHippocampal_R & 28 & -36 & -13 \\
\hline & & Lingual_R & 29 & -44 & -8 \\
\hline & & Temporal_Inf_R & 44 & -42 & -20 \\
\hline \multirow[t]{2}{*}{ Right Postcentral } & \multirow[t]{2}{*}{83} & Postcentral_R & 26 & -38 & 46 \\
\hline & & SupraMarginal_R & 28 & -40 & 44 \\
\hline
\end{tabular}

coefficients of $\mathrm{x}$ and $\mathrm{y}$ and $\mathrm{ss}^{\prime}$ denotes the brain-based similarity structure generated by the label assignment to be scored:

$\mathrm{SCORE}=\mathrm{r}\left(\mathrm{LMOG}_{s s^{\prime}}, I m_{S S}\right) / 2+\mathrm{r}\left(\mathrm{LVTC}_{s s^{\prime}}, I_{s s}\right) / 2+\mathrm{r}\left(\mathrm{LMTG}_{S S^{\prime}}, T x_{S S}\right) / 4$

The weights of $1 / 2$ and $1 / 4$ were chosen without tuning to give Im and Tx equal importance.

Decoding results at the four respective voxel scales were $\{7,7,7,8\}$ (all $\mathrm{p}<.0001$ ). It follows that if we had been given the brain data set blind, and attempted to match the unknown class labels to our known computational model class labels, we would have accurately recovered at least 7/10 classes. In the cases where accuracy was 7/10, errors were as follows: building-part was matched to furniture, furniture to vehicle, and vehicle to building-part. In the 8/10 case vehicle and furniture were confused. To verify that these results were specific to our hypothesized model/brain modality pairings, a set of control tests were run, firstly reversing $I m_{s s}$ and $T x_{S S}$ (so that $I m_{s s}$ was matched with ROIs hypothesized to have stronger associations with $T x_{s s}$ and vice versa) and secondly using either the image-based or text-based model alone to decode all ROIs. Results are in Table 4. None of the control tests were significant. A selection of simpler tests decoding brain region pairs (e.g. LVTC and LMTG; LVTC) that mirror this trend are in Supplementary Materials 3 Table S8.
Table 3

t3.1

Text-based model word-level searchlight: Breakdown of AAL regions in significantly cor- t 3.2 related searchlight clusters (sampled at $1 \mathrm{~mm}^{3}$ ) and MNI coordinates of peak correlations t3.3 per ROI (AAL regions identified as per Just et al., 2010).

\begin{tabular}{|c|c|c|c|c|c|c|}
\hline Cluster & $\begin{array}{l}\text { Vol } \\
\left(\mathrm{mm}^{3}\right)\end{array}$ & ROI & $\mathrm{x}$ & $\mathrm{y}$ & z & $\mathrm{t} 3.5$ \\
\hline \multirow{8}{*}{$\begin{array}{l}\text { Bilateral } \\
\text { Occipital/Parietal/Temporal }\end{array}$} & 838 & Precuneus_L & -17 & -53 & 14 & $\mathrm{t} 3.6$ \\
\hline & & Calcarine_L & -18 & -59 & 13 & $\mathrm{t} 3.7$ \\
\hline & & Cuneus_L & -17 & -55 & 22 & $\mathrm{t} 3.8$ \\
\hline & & Lingual_L & -8 & -67 & 6 & $\mathrm{t} 3.9$ \\
\hline & & Precuneus_R & 16 & -56 & 16 & $\mathrm{t} 3.10$ \\
\hline & & Calcarine_R & 12 & -58 & 18 & $\mathrm{t} 3.11$ \\
\hline & & Cuneus_R & 12 & -58 & 20 & $\mathrm{t} 3.12$ \\
\hline & & Lingual_R & 11 & -61 & 7 & $\mathrm{t} 3.13$ \\
\hline \multirow[t]{2}{*}{ Left Occipital/Parietal } & 252 & Occipital_Mid_L & -43 & -80 & 27 & $\mathrm{t} 3.14$ \\
\hline & & Angular_L & -44 & -78 & 30 & $\mathrm{t} 3.15$ \\
\hline \multirow[t]{3}{*}{ Left Inferior Parietal } & 388 & SupraMarginal_L & -50 & -27 & 30 & $\mathrm{t} 3.16$ \\
\hline & & Parietal_Inf_L & -48 & -31 & 36 & $\mathrm{t} 3.17$ \\
\hline & & Postcentral_L & -53 & -24 & 29 & $\mathrm{t} 3.18$ \\
\hline \multirow[t]{3}{*}{ Left Posterior Parietal } & 113 & Parietal_Inf_L & -30 & -55 & 40 & $\mathrm{t} 3.19$ \\
\hline & & Angular_L & -35 & -55 & 36 & $\mathrm{t} 3.20$ \\
\hline & & Parietal_Sup_L & -32 & -60 & 44 & $\mathrm{t} 3.21$ \\
\hline \multirow[t]{5}{*}{ Left Ventral Temporal } & 1200 & Fusiform_L & -32 & -39 & -19 & $\mathrm{t} 3.22$ \\
\hline & & Cerebelum_4_5_L & -29 & -42 & -24 & $\mathrm{t} 3.23$ \\
\hline & & Cerebelum_6_L & -31 & -44 & -24 & $\mathrm{t} 3.24$ \\
\hline & & ParaHippocampal_L & -30 & -39 & -11 & $\mathrm{t} 3.25$ \\
\hline & & Temporal_Inf_L & -36 & -37 & -15 & $\mathrm{t} 3.26$ \\
\hline \multirow[t]{2}{*}{ Left Lateral Temporal } & 236 & Temporal_Inf_L & -59 & -56 & -16 & $\mathrm{t} 3.27$ \\
\hline & & Temporal_Mid_L & -50 & -60 & -4 & $\mathrm{t} 3.28$ \\
\hline \multirow[t]{3}{*}{ Right Ventral Temporal } & 321 & Fusiform_R & 31 & -33 & -18 & $\mathrm{t} 3.29$ \\
\hline & & ParaHippocampal_R & 29 & -29 & -18 & $\mathrm{t} 3.30$ \\
\hline & & Cerebelum_4_5_R & 28 & -35 & -24 & $\mathrm{t} 3.31$ \\
\hline \multirow[t]{4}{*}{ Left Inferior Frontal } & 78 & Frontal_Inf_Oper_L & -50 & 10 & 16 & $\mathrm{t} 3.32$ \\
\hline & & Precentral_L & -50 & 6 & 14 & $\mathrm{t} 3.33$ \\
\hline & & Frontal_Inf_Tri_L & -48 & 12 & 24 & $\mathrm{t} 3.34$ \\
\hline & & Frontal_Inf_Tri_L & -48 & 12 & 24 & $\mathrm{t} 3.35$ \\
\hline
\end{tabular}

Summarizing, we achieved high-accuracy decoding of brain repre- 936 sentations in an object-selective visual area using our image-based 937 model. This decoding was not possible using the text-based model. 938 We claim that this is evidence that rich visually-embodied object repre- 939 sentations are induced by thought in lack of an overt visual stimulus. 940 Next, we showed that we could make results more robust by exploiting 941 multimodal models with a novel adaptation of the decoding algorithm. 942 A decoding test directly encapsulating our original modal-specificity hy- 943 potheses had accuracy between $7 / 10$ and 8/10 (consistent with our pre- 944 dictions of image and text-based model dominance in different brain 945 regions).

\section{Discussion}

947

We tested whether fMRI activity patterns elicited by participants 948 reading object names (without visual cues) incorporate embodied visu- 949 al representations of the objects, and whether we could decode the ob- 950 ject class from the representation. To test for visually embodied 951 representations we used novel image-based models derived from object 952 features in conjunction with text-based models to distinguish visual/ 953 non-visual aspects of the brain's semantic representation. Different to 954 all previous studies, our image-based models were not modeling a spe- 955 cific visual stimulus, but more generic imagery related to a concept. $\quad 956$

Significantly greater correlation with image-based models was ob- 957 served in visual object-selective brain regions, and with text-based 958 models in brain areas posited to be modality independent. We further 959 demonstrated that the image-, but not the text-based models, could de- 960 code object classes from visual brain representations with high accura- 961 cy, and, when we applied our models in combination to decode different 962 brain regions in parallel, we got consistently high decoding accuracies. 963 Key points are: (1) This provides evidence that rich embodied visual 964 representations of objects are induced as words are read and contem- 965 plated. This mental imagery implicit in conceptual fMRI data contains 966 
t4.1 Table 4

(the incon-

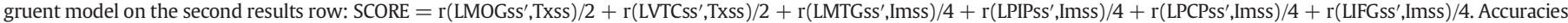
$\geq 3$ (in bold) are significant ( $\mathrm{p}<.05$, Binomial test). Further tests with model/ROI pairs are in Table S8.

\begin{tabular}{|c|c|c|c|c|c|c|c|c|c|}
\hline $\mathrm{LMOG}_{s s^{\prime}}$ & LVTC $_{s s^{\prime}}$ & $\mathrm{LMTG}_{s s^{\prime}}$ & $\mathrm{LIFG}_{s s^{\prime}}$ & $\mathrm{LPIP}_{s s^{\prime}}$ & $\mathrm{LPCP}_{s s^{\prime}}$ & $50 \mathrm{vox}$ & 100vox & 200vox & 400vox \\
\hline$I m_{s s}$ & $I m_{s s}$ & $T x_{s S}$ & $T x_{s s}$ & $T x_{s S}$ & $T x_{s s}$ & 7 & 7 & 7 & 8 \\
\hline$T x_{S S}$ & $T x_{s s}$ & $I m_{s s}$ & $I m_{s s}$ & $I m_{s s}$ & $I m_{s s}$ & 0 & 0 & 0 & 0 \\
\hline$I m_{s S}$ & $I m_{s s}$ & $I m_{s s}$ & $I m_{s s}$ & $I m_{s s}$ & $I m_{s s}$ & 0 & 0 & 1 & 1 \\
\hline$T x_{s s}$ & $T x_{s s}$ & $T x_{s s}$ & $T x_{s s}$ & $T x_{s s}$ & $T x_{s s}$ & 0 & 0 & 0 & 0 \\
\hline
\end{tabular}

967 more detailed visual categorical information than has previously been established (Pulvermüller, 2013) even in rehearsed visual imagery where fewer object categories have been successfully discriminated (Lee et al., 2012; Reddy et al., 2010) and is in line with the perceptual representations/mental simulations posited by Paivio (1971), Glaser (1992), Barsalou et al. (2008), Barsalou, 2009; (2) Visual semantic representations of objects are likely to be embodied in lower-level features than are amenable to linguistic representation in line with the depictive view of imagery (Kosslyn and Thompson, 2003) and also Lee et al. (2012); (3) Computational visual models can decode internally induced embodied representations. (4) We bring support to hypotheses derived from recent literature of how brain regions differentially contribute to encode semantic information.

What is the similarity between image-based models and brain representations?

Similarity in sensory input - we sourced the image-based models from a diverse selection of snapshots of objects in natural scenes that might approximate participants' ecological experience.

Similarity in representational features - that words provide a viable basis set for linguistic semantic representations, as per the text-based models, is not difficult to argue. Conversely, our dictionary of visual featuress, which were abstract combinations of local image descriptors was estimated empirically from an image collection. As can be seen from the backprojection of visual features onto natural scenes in Fig. 2, although visual features cluster on apparently similar local visual patterns, and there is evidence of systematic associations between them and classes of depicted objects, it is not straightforward to interpret what each visual feature represents. Obviously, it would be nice to have a neatly interpretable set of visual features, that could be easily defined in parameters such as shape, color and texture, similar to artificial stimuli used to probe visual object representations (e.g., Drucker and Aguirre, 2009; Op de Beeck et al., 2008). However, in hand with the suggestion that visually embodied representations may be grounded in lower level features than are amenable to linguistic description, it may not be easy to verbally define low-level embodied brain features. The computational tools used to build the image-based models draw upon an extensive history of computer vision research attempting to devise algorithms that are robust enough to deal with real world object recognition applications. Similar task demands (e.g., template matching in attention and mental imagery in planning) were presumably fundamental to shape biological concept representations. We thus conjecture that robustness constraints may have streamlined similar computational properties in both biological and artificial models.

Similarity in distributional representation (of features) - Demonstrating that neural object representations are spatially distributed was a founding step for multi-voxel pattern analyses (Haxby et al., 2001) and the principle of feature co-occurrence, used to derive computational semantic models, is akin to Hebbian learning. Assuming that neural associations are formed between combinations of nodes (neural populations receptive to shape fragments/shapes/words) that are frequently co-activated, then co-occurrence constitutes a valid approximation.

The validity of these assertions is amenable to future experimental testing through systematically modifying the diversity of information in source data, visual feature extraction strategies and nature of distrib- 1020 uted representation, and comparing the fit to brain data.

Implications for the empirical study of embodied concept representations

An ability to interpret latent visual structure in brain representations 1023 adds credibility to an experimental approach complementary to the 1024 current "tradition emphasizing stimulus driven brain activity" (Binder Q16 and Desai, 2011). Contrary to perceptual experiments that aim to iden- 1026 tify brain activity that covaries with controlled change in physical stim- 1027 ulus properties, studies of semantic memory aim to explain brain 1028 patterns derived from past perceptual experience. Computational se- 1029 mantic models, as we have shown, can provide a route into the study 1030 of internally induced and modality-preferential brain representations 1031 that are difficult to interpret otherwise (for instance embodied repre- 1032 sentations arising from dreaming). Additionally in much the same 1033 way as 'the book was better than the film', we conjecture that verbal 1034 stimuli may provide the best default means to trigger rich semantic rep- 1035 resentations even in their visual aspects. It follows that presenting spe- 1036 cific modal information (e.g., specific dog pictures) in stimuli designed 1037 to probe general semantic representations may paradoxically limit valu- 1038 able semantic content extracted from memory, by focusing the neural 1039 representation on the specific stimulus.

Implications for computational models of conceptual knowledge

(1) There is by now a long line of studies showing that computation- 1042 al models encoding statistical generalizations extracted from 1043 large bodies of text can simulate various aspects of human se- 1044 mantic memory (see, e.g., discussion and references in Lenci, 1045 2008). Given the concomitant success of embodied approaches 1046 to meaning, a natural question arises about the division of 1047 labor, in grounding conceptual knowledge, between linguistic 1048 statistics and situated knowledge (e.g., Barsalou et al., 2008; 1049 Louwerse, 2008). As part of this debate, various authors have de- 1050 veloped computational models that use subject-generated con- 1051 cept property descriptions as proxies to embodied experiential 1052 data (e.g., Andrews et al., 2009; Johns and Jones, 2012). While 1053 these simulations provide good insights into how the two knowl- 1054 edge sources can be integrated, subject-generated word lists are 1055 a rather artificial (and ultimately linguistic!) surrogate of percep- 1056 tual knowledge. Our multimodal decoding experiments also 1057 confirm that integrating perceptual (specifically, visual) and lin- 1058 guistic information provides more human-like semantic repre- 1059 sentations. However, we approximate perceptual information 1060 with a model that is genuinely not verbal, but induced from nat- 1061 ural images, combined with a state-of-the-art linguistic model. 1062 We thus pave the way to more realistic simulations of how lin- 1063 guistic and perceptual evidence are integrated into human con- 1064 ceptual knowledge.

(2) Brain data provides a useful test-bed for evaluating multimodal 1066 semantic models. The traditional approach to appraise semantic 1067 models compares human similarity judgments to models. As 1068 models incorporate additional modal information it becomes dif- 1069 ficult to know how to configure norming questions appropriate- 1070 ly. Even with only vision there are many ways to measure 1071 


\section{Acknowledgments}

Marcel Just for generously providing fMRI data for reanalysis, two 1081 anonymous reviewers for their useful suggestions and Rajeev Raizada 1082 for commentary on the curse of knowledge.

\section{Appendix A. Supplementary data}

1084

Supplementary data to this article can be found online at http://dx 1085 doi.org/10.1016/j.neuroimage.2015.06.093.

\section{References}

Anderson, A.J., Bruni, E., Bordignon, U., Poesio, M., Baroni, M., 2013. Of words, eyes and brains: Correlating image-based distributional semantic models with neural representations of concepts. Proceedings of EMNLP 2013 1960-1970 (Seattle, WA).

Andrews, M., Vigliocco, G., Vinson, D., 2009. Integrating experiential and distributional data to learn semantic representations. Psychol. Rev. 116 (3), 463-498.

Barsalou, L.W., 2009. Simulation, situated conceptualization, and prediction. Philos. Trans. R. Soc. Lond. B Biol. Sci. 364, 1281-1289.

Barsalou, L.W., Santos, A., Simmons, W.K., Wilson, C.D., 2008. Language and simulation in conceptual processing. In: De Vega, M., Glenberg, A.M., Graesser, A.C. (Eds.), Symbols, embodiment, and meaning. Oxford University Press, Oxford, pp. 245-283.

Bedny, M., Caramazza, A., 2011. Perception, action, and word meanings in the human brain: the case from action verbs. Ann. N. Y. Acad. Sci. 1224, 81-95.

Binder, J.R., Desai, R.H., 2011. The neurobiology of semantic memory. Trends Cogn. Sci. 15 , 527-536.

Binder, J.R., Desai, R.H., Graves, W.W., Conant, L.L., 2009. Where is the semantic system? A critical review and meta-analysis of 120 functional neuroimaging studies. Cereb. Cortex 19, 2767-2796.

Bruffaerts, R., Dupont, P., Peeters, R., De Deyne, S., Storms, G., Vandenberghe, R., 2013. Similarity of fMRI activity patterns in left perirhinal cortex reflects similarity between words. J. Neurosci. 33 (47), 18597-18607.

Bruni, E., Bordignon, U., Liska, A., Uijlings, J., Sergienya, I., 2013. Vsem: An open library for visual semantics representation. Proceedings of ACL, pp. 187-192 (Sofia, Bulgaria).

Bruni, E., Tran, N., Baroni, M., 2014. Multimodal distributional semantics. J. Artif. Intell. Res. 49, 1-47.

Carlson, T.A., Simmons, R.A., Kriegeskorte, N., Slevc, L.R., 2014. The emergence of semantic meaning in the ventral temporal pathway. J. Cogn. Neurosci. 26 (1), 120-131.

Chatfield, K., Lempitsky, V., Vedaldi, A., Zisserman, A., 2011. The devil is in the details: an evaluation of recent feature encoding methods. Proceedings of BMVC.

Church, K., Hanks, P., 1990. Word association norms, mutual information, and lexicography. Comput. Linguist. 16 (1), 22-29 (DC).

Clark, S., 2013. Vector space models of lexical meaning. In: Lappin, S., Fox, C. (Eds.), Handbook of contemporary semantics, 2nd ed. Blackwell, Malden, MA.

Connell, L., Lynott, D., 2014. Principles of representation: why you can't represent the same concept twice. Top. Cogn. Sci. 6 (3), 390-406.

Connolly, A.C., Guntupalli, J.S., Gors, J., Hanke, M., Halchenko, Y.O., Wu, Y.C., et al., 2012. The representation of biological classes in the human brain. J. Neurosci. 32, 2608-2618.

Csurka, G., Dance, C., Fan, L., Willamowski, J., Bray, C., 2004. Visual categorization with bags of keypoints. Workshop on statistical learning in computer vision. ECCV, pp. $1-22$.

Deng. J., Dong, W., Socher, R., Li, L., Fei-Fei, L., 2009. Imagenet: A large-scale hierarchical image database. Proceedings of CVPR, pp. 248-255 (Miami Beach, FL).

Devereux, B., Kelly, C., Korhonen, A., 2010. Using fMRI activation to conceptual stimuli to evaluate methods for extracting conceptual representations from corpora. In: Murphy, B., Chang, K.K., Korhonen, A. (Eds.), Proceedings of the NAACL HLT 2010 First Workshop on Computational Neurolinguistics. Association for Computational Linguistics, Los Angeles, USA, pp. 70-78.

Devereux, B.J., Clarke, A., Marouchos, A., Tyler, L.K., 2013. Representational similarity analysis reveals commonalities and differences in the semantic processing of words and objects. J. Neurosci. 33 (48), 18906-18916.

Drucker, D.M., Aguirre, G.K., 2009. Different spatial scales of shape similarity representation in lateral and ventral LOC. Cereb. Cortex 19 (10), 2269-2280.

Evert, S., 2005. The Statistics of Word Cooccurrences. Ph.D dissertation, Stuttgart University.

Fairhall, S., Caramazza, A., 2013. Brain regions that represent amodal conceptual knowledge. J. Neurosci. 33, 10552-10558.
Genovese, C.R., Lazar, N.A.. Nichols, T., 2002. Thresholding of statistical maps in functional 1144 neuroimaging using the false discovery rate. Neurolmage 15 (4), 870-878. Glaser, W.R., 1992. Picture naming. Cognition 42, 61-105. 1146

Grauman, K., Darrell, T., 2005. The pyramid match kernel: Discriminative classification Q22 with sets of image features. Proceedings of ICCV, pp. 1458-1465 (Beijing, China). 1148

Grauman, K., Leibe, B., 2011. Visual object recognition. Morgan \& Claypool, San Francisco. 1149

Hassabis, D., Kumaran, D., Maguire, E.A., 2007. Using imagination to understand the neu- 1150 ral basis of episodic memory. J. Neurosci. 27 (52), 14365-14374. 1151

Hauk, O., Johnsrude, I., Pulvermüller, F., 2004. Somatotopic representation of action words 1152 in human motor and premotor cortex. Neuron 41 (2), 301-307. 1153

Haxby, J.V., Gobbini, M.I., Furey, M.L., Ishai, A., Schouten, J.L., Pietrini, P., 2001. Distributed 1154 and overlapping representations of faces and objects in ventral temporal cortex. Sci- 1155 ence 293, 2425-2430.

1156

Hiramatsu, C., Goda, N., Komatsu, H., 2011. Transformation from image-based to percep- 1157 tual representation of materials along the human ventral visual pathway. 1158 Neurolmage 57 (2), 482-494. 1159

Ishai, A., Ungerleider, L.G., Haxby, J.V., 2000. Distributed neural systems for the generation 1160 of visual images. Neuron 28 (3), 979-990. 1161

Johns, B., Jones, M., 2012. Perceptual inference through global lexical similarity. Top. Cogn. 1162 Sci. 4 (1), 103-120.

Just, M.A., Cherkassky, V.L., Aryal, S., Mitchell, T.M., 2010. A neurosemantic theory of con- 1164 crete noun representation based on the underlying brain codes. PLoS ONE 5 (1), 1165 e8622. 1166

Kellenbach, M.L., Wijers, A.A., Mulder, G., 2000. Visual semantic features are activated 1167 during the processing of concrete words: Event-related potential evidence for per- 1168 ceptual semantic priming. Cogn. Brain Res. 10 (1), 67-75. 1169

Khaligh-Razavi, S.-M., Kriegeskorte, N., 2014. Deep supervised, but not unsupervised 1170 models may explain IT cortical representation. PLoS Comput. Biol. 10 (11), 1171 e1003915. http://dx.doi.org/10.1371/journal.pcbi.1003915. 1172

Kiefer, M., Sim, E.-J., Herrnberger, B., Grother, J., Hoenig, K., 2008. The sound of concepts: 1173 Four markers for a link between auditory and conceptual brain systems. J. Neurosci. 1174 28 (47), 12224-12230. 1175

Kosslyn, S.M., Thompson, W.L., 2003. When is early visual cortex activated during visual 1176 mental imagery? Psychol. Bull. 129, 723-746.

1177

Krause, B.J., Schmidt, D., Mottaghy, F.M., Taylor, J., Halsband, U., Herzog, H., Tellmann, L., 1178 Müller-Gärtner, H.-W., 1999. Episodic retrieval activates the precuneus irrespective 1179 of the imagery content of word pair associates. Brain 122 (2), 255-263. http://dx. 1180 doi.org/10.1093/brain/122.2.255.

Kriegeskorte, N., Goebel, R., Bandettini, P., 2006. Information-based functional brain map- 1182 ping. Proc. Natl. Acad. Sci. U. S. A. 103, 3863-3868. 1183

Kriegeskorte, N., Mur, M., Bandettini, P., 2008a. Representational similarity analysis - 1184 Connecting the branches of systems neuroscience. Front. Syst. Neurosci. 2 (4), 1185 $1-28$.

Kriegeskorte, N., Mur, M., Ruff, D.A., Kiani, R., Bodurka, J., Esteky, H., Tanaka, K., Bandettini, 1187 P.A., 2008b. Matching categorical object representations in inferior temporal cortex of 1188 man and monkey. Neuron 60, 1126-1141. 1189

Landauer, T., Dumais, S., 1997. A solution to Plato's problem: The latent semantic analysis 1190 theory of acquisition, induction, and representation of knowledge. Psychol. Rev. 1041191 (2), 211-240.

Lazebnik, S., Schmid, C., Ponce, J. 2006. Beyond bags of features: Spatial pyramid Q23 matching for recognizing natural scene categories. Proceedings of CVPR, 1194 pp. 2169-2178 (Washington). 1195

Lee, S.H., Kravitz, D.J., Baker, C.I., 2012. Disentangling visual imagery and perception of 1196 real-world objects. NeuroImage 59, 4064-4073.

Leeds, D.D., Seibert, D.A., Pyles, J.A., Tarr, M.J., 2013. Comparing visual representations Q24 across human fMRI and computational vision. J. Vis. 13 (13), 1-27 (25). 1199

Lenci, A., 2008. Distributional semantics in linguistic and cognitive research. Ital. 1200 J. Linguist. 20 (1), 1-31.

Louwerse, M., 2008. Embodied representations are encoded in language. Psychon. Bull. 1202 Rev. 15, 838-844. 1203

Louwerse, M.M., Hutchinson, S., 2012. Neurological evidence linguistic processes precede 1204 perceptual simulation in conceptual processing. Front. Psychol. 3, 385. http://dx.doi. 1205 org/10.3389/fpsyg.2012.00385. 1206

Lowe, D., 2004. Distinctive image features from scale-invariant keypoints. Int. J. Comput. 1207 Vis. 60 (2), 91-110.

Lund, K., Burgess, C., 1996. Producing high-dimensional semantic spaces from lexical co- 1209 occurrence. Behav. Res. Methods Instrum. Comput. 28, 203-208. 1210

Mahon, B.Z., Caramazza, A., 2009. Concepts and categories: a cognitive neuropsychologi- 1211 cal perspective. Annu. Rev. Psychol. 60, 27-51. 1212

Manning, C., Schütze, H., 1999. Foundations of statistical natural language processing. MIT 1213 press.

Manning, C., Raghavan, P., Schütze, H., 2008. Introduction to information retrieval. Cam- 1215 bridge University Press, Cambridge, UK. 1216

Martin, A., 2007. The representation of object concepts in the brain. Annu. Rev. Psychol. 1217 $58,25-45$.

Mitchell, T.M., Shinkareva, S.V., Carlson, A., Chang, K.-M., Malave, V.L., Mason, R.A., Just, 1219 M.A., 2008. Predicting human brain activity associated with the meaning of nouns. 1220 Science 320, 1191-1195.

Murphy, B., Talukdar, P., Mitchell, T., 2012. Selecting corpus-semantic models for 1222 neurolinguistic decoding. Proceedings of First Joint Conference on Lexical and Com- 1223 putational Semantics ( ${ }^{*}$ SEM $)$, p. 114.

O'Craven, K. Downing P. Kanwisher, N 1999. fMRI evidence for objects as the units of attentional selection. Nature 401, 584-587.

Op de Beeck, H.P., Torfs, K., Wagemans, J., 2008. Perceived shape similarity among unfa- 1226 miliar objects and the organization of the human object vision pathway. J. Neurosci. 1228 28, 10111-10123. 
Paivio, A., 1971. Imagery and verbal processes. Holt, Rinehart, and Winston, New York Peelen, M.V., Fei-fei, L., Kastner, S., 2009. Neural mechanisms of rapid natural scene categorization in human visual cortex. Nature 460, 94-97.

Pulvermüller, F., 2013. How neurons make meaning: brain mechanisms for embodied and abstract-symbolic semantics. Trends Cogn. Sci. 17 (9), 458-470.

Pulvermüller, F., Cooper-Pye, E., Dine, C., Hauk, O., Nestor, P.J., Patterson, K., 2010. The word processing deficit in semantic dementia: all categories are equal, but some categories are more equal than others. J. Cogn. Neurosci. 22 (9), 2027-2041.

Raizada, R.D.S., Connolly, A.C., 2012. What makes different people's representations alike: neural similarity-space solves the problem of across-subject fMRI decoding. J. Cogn. Neurosci. 24 (4), 868-877.

Reddy, L., Tsuchiya, N., Serre, T., 2010. Reading the mind's eye: decoding category information during mental imagery. NeuroImage 50, 818-825.

Serre, T., Oliva, A., Poggio, T., 2007. A feedforward architecture accounts for rapid categorization. Proc. Natl. Acad. Sci. U. S. A. 104, 6424-6429.

Sivic, J., Zisserman, A., 2003. Video Google: A text retrieval approach to object matching in videos. Proceedings of ICCV, pp. 1470-1477 (Nice, France).

Steiger, J.H., 1980. Tests for comparing elements of a correlation matrix. Psychol. Bull. 87 (2), 245-251.

Stokes, M., Thompson, R., Cusack, R., Duncan, J., 2009a. Top-down activation of shapespecific population codes in visual cortex during mental imagery. J. Neurosci. 29 1565-1572.
Stokes, M., Thompson, R., Nobre, A.C., Duncan, J., 2009b. Shape-specific preparatory activ- 1252 ity mediates attention to targets in human visual cortex. Proc. Natl. Acad. Sci. U. S. A. 1253 106, 19569-19574. 1254

Stokes, M., Saraiva, A., Rohenkohl, G., Nobre, A.C., 2011. Imagery for shapes activates 1255 position-invariant representations in human visual cortex. NeuroImage 56, 1256 1540-1545. 1257

Trumpp, N.M., Kliese, D., Hoenig, K., Haarmeier, T., Kiefer, M., 2013. Losing the sound of 1258 concepts: Damage to auditory association cortex impairs the processing of sound- 1259 related concepts. Cortex 49 (2), 474-486. 1260

Turney, P., Pantel, P., 2010. From frequency to meaning: Vector space models of seman- 1261 tics. J. Artif. Intell. Res. 37, 141-188. 1262

Tzourio-Mazoyer, N., Landeau, B., Papathanassiou, D., Crivello, F., Etard, O., Delcroix, N., 1263 Mazoyer, B., Joliot, M., 2002. Automated anatomical labeling of activations in SPM 1264 using a macroscopic anatomical parcellation of the MNI MRI single-subject brain. 1265 Neurolmage 15, 273-289.

Yanulevskaya, V., Uijlings, J., Bruni, E., Sartori, A., Zamboni, E., Bacci, F., Melcher, D., Sebe, 1267 N., 2012. In the eye of the beholder: employing statistical analysis and eye tracking 1268 for analyzing abstract paintings. Proceedings of the 20th ACM international conference on Multimedia. ACM, pp. 349-358.

Zwaan, R.A., Stanfield, R.A., Yaxley, R.H., 2002. Language comprehenders mentally represent the shapes of objects. Psychol. Sci. 13 (2), 168-171. 